\title{
Catálogo de Incunables de la biblioteca del Colegio de PP. Agustinos Filipinos de Valladolid
}

Empeñados en la ardua tarea de catalogar, según criterios modernos, los libros existentes en la citada Biblioteca, hemos querido comenzar con los incunables. A parte de esporádicas menciones ${ }^{1}$, puede decirse que solamente el indice de los Incunables de autores agustinos es conocido ${ }^{2}, \mathrm{y}$, por cierto, no mucho ${ }^{3}$.

Nos ha movido a la publicación de este catálogo el hecho de que esté en elaboración el Catálogo colectivo de Incunables existentes en las Bibliotecas Españolas "Esta obra tiene un carácter provisional: consta de tres volúmenes policopiados, destinados a recoger las observaciones de las bibliotecas; el deseo es que la edición definitiva recoja todos los Incunables existentes en España, a ejemplo de 10 que han hecho otras naciones.

1. Procedencia de los Incunables.

La historia de la Biblioteca no es muy larga 5. Podriamos decir

1. L. GARCIA, "Nuestra Biblioteca": Casiciaco 3 (1949) 193. En esta entrevista el P. Francisco Aymerich habla de 92 Incunables existentes en la Biblioteca; D. RODRIGUEZ, "El colegio y la ciudad de Valladolid", Archivo Agustiniano 53 (1959) 179. dice que son "más de ciento" los Incunables de esta Biblioteca.

2. A. BLANCO, Biblioteca bibliográfico-agistiniana del Colegio de Valladolid. Valladolid, 1909, publicó los Incunables de autores agustinos que existían entonces en la Biblioteca. Más recientemente C. ALONSO, "Incunables agustinianos en nuestra Biblioteca de Valladolid": Casiciaco 6 (1952) 298-300, publicó el índice de los Incunables de autores agustinos:

3. F. CANTELAR RODRIGUEZ, "Catálogo de incunables en bibliotecas españolas.". Repertorio de las Ciencias Eclesiásticas en España, 5 : Siglos I-XVI (Salamanca 1976) 507-531, hace una larga reseña de las bibliotecas españolas en las que existen Incunables y no enumera la de los Agustinos de Valladolid.

4. La Dirección General de Archivos y Bibliotecas ha publicado el Catálogo colectivo provisional de Incunables existentes en las Bibliotecas Españolas (Madrid, s. a.). (3 vols.). Policopiados. Reseña 4.852 obras y tiene un apéndice con 116 obras que no aparecen descritas en los repertorios más conocidos.

5. En A. BLANCO, Biblioteca bibliográfico-agustiniana, LXV-LXXXIX, puede leerse un resumen de la historia de la Biblioteca. 
que nace con el Colegio, a mediados del s.XVIII, muy lejos, pues, de la época de los Incunables. Estos han venido a parar a esta biblioteca procedentes de varias adquisiciones. La mayor parte tiene una procedencia inmediata perfectamente conocida. De las 125 piezas que vamos a catalogar, proceden 99 de Shang-hai (China), de nuestra Casa-Procuración de aquella ciudad. Todos ellos tienen el sello de dicha Casa. Fueron adquiridos por el P. Gaudencio Castrillo hacia el año 1920 de un negociante alemán, a quien la Provincia había prestado dinero y pagó sus deudas mediante estos Incunables. ¿Cómo tales Incunables fueron a parar a manos del negociante alemán? Todos ellos llevan, aún hoy, pegado en su cubierta interior un papelito de catálogo de un Antiquariado, que ofrece una descripción somera del ejemplar y cita el número correspondiente del repertorio de Hain. Todos ellos, pues, proceden de una Libreria-Antiquariado, ciertamente alemana, y, con toda probabilidad, de Leipzig, que vendía tales ejemplares.

Esta Casa-Antiquariado debió de adquirirlos de varios conventos pertenecientes a diversas ordenes religiosas y otras Instituciones. La mayor parte, en efecto; tiene algunas notas manuscritas; que indican el convento a donde pertenecian o alguna nota "ex-libris", con los nombres de los antiguos propietarios de los Incunables. En su mayoría proceden de Alemania. Indicaremos, en este catálogo, cuáles son estos Incunables mediante un asterisco, delante del número de orden.

Las 26 piezas restantes tienen una procedencia variada. Dos proceden de nuestra Casa de Manila, los números 5 y 51. Otros dos, los números 34 y 63 proceden del Convento de Agustinos de Sarria (Lugo). Es muy probable que llegaran a esta Biblioteca en tiempos de la desamortización. Se sabe que el P. Francisco Villacorta, Comisario-Procurador de la Provincia de Filipinas ante las Cortes de Madrid y Roma (1818-1843), recorrió diversos conventos de Agustinos, adquiriendo libros, ya que este Colegio-Seminario se vio libre de tal medida gubernamental. Quizá también el número 22 fue adquirido en la misma época, procedente del Convento de S. Agustín de Valladolid y que pertenecía al P. Corral, agustino de la Provincia de Castilla, cuyos libros fueron adquiridos por esta Biblioteca. Otros, cuya procedencia se ignora (como los números $30,64,104$ y 105) y algunos más que tienen la nota manuscrita: "Perteneciente a la Libreria de los PP. Agustinos-Filipinos de Valladolid" (como los números $35,95,98,117$ y 118), quizá fueron adquiridos en las aludidas circunstancias. Finalmente, hay algunos como los números $1,2,106$ y 69 que proceden de Italia. Los tres primeros, probable- 
mente, fueron adquiridos por el $\mathbf{P}$. Agustín Oña, Comisario-Procurador de la Provincia de Filipinas en Roma, de quien se sabe envió numerosos cajones de libros comprados allá para esta Biblioteca. El último número citado fue adquirido en Roma en 1970.

\section{Carácter de esta Colección.}

Dada la procedencia de los Incunables, es fácil intuir que no se trata de una colección homogénea. Los Incunables se adquirieron por ser Incunables, no por tratar de esta o aquella materia. Quizá solamente el P. Agustín Oña compró los que hemos indicado porque eran Incunables de autores agustinos.

De hecho hay obras de todas las clases: Biblias, obras teológicas como las de Pedro Lombardo, Tomás de Aquino, Duns Scoto, Antonino Florentino etc.; filosóficas, como las de Egidio Romano, Alfonso Vargas de Toledo, Alberto de Sajonia, Pablo Véneto etc.; jurídicas, como las Decretales y las obras de Azo Portius, Francisco de Zabarellis, etc. clásicas y de literatura, como las obras de Quintiliano, Marcial, Lucano, Ovidio, Petrarca, etc.

Hay alguno valioso por su antigüedad, como el número 80 , que es de 1472, aunque en general predominan los impresos en la década de los ochenta y noventa. Como particularmente raros, hacemos notar el número 69 que solamente hemos encontrado en Goff y el número 102 que no hemos visto en los repertorios más corrientes.

Dada la procedencia de la mayor parte de los Incunables, no es extraño que no haya ninguno que esté impreso en España. Predominan los impresos en Venecia, como es natural, aunque están bien representadas las ciudades alemanas de Nüremberg, Basilea $y$ Estrasburgo.

\section{Caracteristicas de este Catálogo}

Seguiremos en este catálogo el orden alfabético de autores, porque lo creemos de mayor utilidad. Haremos los necesarios reenvios, para que sea fácil encontrar determinada obra.

Reseñamos todos los Incunables de la Biblioteca, aunque pudiera darse el caso, que quedara alguno todavía mezclado entre los demás libros de la Biblioteca.

Tomamos el término Incunable en su acepción estricta, es decir, los libros impresos antes de 1500 inclusive. No catalogaremos, pues, 
los libros impresos en los primeros lustros del s. XVI, aunque sabemos que esta división es puramente convencional y no responde a una realidad tipográfica, y de lo cual hay buenos ejemplos en esta biblioteca. Por ejemplo el número 1, que es de 1500, está encuadernado con otra obra de Egidio Romano, que es de 1502 ; los dos proceden de la misma imprenta y no hay diferencia tipográfica alguna entre ellos.

La descripción que hacemos es la asi llamada "semiplena" ", que ordinariamente es suficiente para individuar la obra. Hemos procurado citar los repertorios más conocidos en la medida de 10 posible y reenviar al Catálogo colectivo, cuando allí lo hemos encontrado.

6. Así es llamada esta descripción por los autores siguientes: A. GARCIA Y GARCIA, F. CANTELAR RODRIGUEZ, M. NIETO CUMPLIDO, Catálogo de los Manuscritos e Incunables de la Catedral de Córdoba (Salamanca 1976) LXXIX; F. CANTELAR RODRIGUEZ, Catálogo de Incunables, 508-509. 


\section{Bibliografia y abreviaturas}

ca. $=$ circa.

Catálogo colectivo = DIRECCION GENERAL DE ARCHIVOS Y BIBLIOTECAS, Catálogo colectivo Provisional de Incunables existentes en las Bibliotecas Españolas (Madrid s. f.) 3 vols. policopiados.

col. = columna $(\mathrm{s})$.

Copinger $=W$ A. COPINGER, Supplement to Hain's Repertorium Bibliophraphicum or Collections towards a new edition of that work. London 1895-1902 = Milano 1950).

Córdoba, Cat = A. GARCIA Y GARCIA-F. CANTELAR RODRIGUEZ M. NIETO CUMPLIDO, Catálogo de los Manuscritos e Incunables de la Catedrál de Córdoba. (Salamanca 1976).

Escorial, Agustinos = T. ALONSO TURIENZO, "Indice de Incunables de la Real Biblioteca del Escorial y Biblioteca de la Comunidad de PP. Agustinos", La Ciudad de Dios 187 (1974) 665-668.

Escorial, Monasterio = IDEM, ib. 187 (1974) 646-665.

Esc. tip. = Escudo tipográfico.

Espac. para inic. = Espacios para iniciales.

f. $=$ folio.

ff. $=$ folios

fol. = foliación.

Fol. = Folio (tamaño del Incunable).

$\mathrm{GW}=$ Gesamtkatalog der Wiegendrucke (Leipzig 1925-1940 = Stuttgart-New York 1968).

Goff $=$ F. R. GOFF, Incunabula in American Libraries. A third census of fifteenth century books recorded in North American Collections (New York $1964=$ New York 1973).

gót. = gótica.

gr. = griega.

Hain: L. HAIN, Repertorium bibiiographicum, in quo libri omnes ab arte. typographica inventa usque ad annum MD. typis expressi ordine alphabetico vel simpliciter enumerantur vel adcuratius recensentur (Stuttgartiae et Lutetiae Parisiorum 1826-1838 = Milano 1948).

hoj. $=$ hojas.

Incun. Hungar. = G. SAJO-E. SOLTESZ, Catalogus Incunabulorum quae in bibliothecis Hungariae asservantur. 2 vols. (Budapestini 1970).

Incun. Ital $=$ T. M. GUARNASCHELLI-E. VALENZIANI, Indice Generale degli Incunaboli delle bibliotheche d'Italia. Vol. I: A-B (Roma 1943); vol. II: C-F (Roma 1948); los autores citados con la colaboración de E. CERULLI, vol. III: G-L (Roma 1954); E. VALENZIANI - E. CERULLI, vol. IV: M-R (Roma 1965); estos dos más P. VALENZIANI y A. TINTO, vol. V: S-Z (ROMA 1972). 
Incun. Polon. = Incunabula quae in bibliothecis Poloniae asservantur moderante A. KAWECKA - GRYCZOWA composuerunt M. BOHONOS et E. SZANDOROWSKA (Wratislaviae - Varsaviae - Cracoviae 1970).

Inic. $=$ Iniciales

L. = Letra.

L. gót. = Letra gótica.

L. gr. = Letra griega.

lin. $=$ líneas.

L. rom. = Letra romana.

Madrid BN = D. GARCIA ROJO-G. ORTIZ DE MONTALVAN, Catálogo de Incunables de la Biblioteca Nacional. (Madrid 1945).

Mayúsc. = Mayúsculas.

minúsc. $=$ minúsculas.

P. $=$ Parte.

Pellechet $=$ M. PELLECHET, Catalogue général des incunables des bibliothèques publiques de France. (Paris 1897-1909).

Polain $=$ M. L. POLAIN, Catalogue des livres imprimés au XV siècle des bibliothèques de Belgique (Bruxelles 1932).

Proctor: R. PROCTOR, An Index to the early printed books in the British Museum: from the invention of printing to the year 1500. Woth notes of those in the Bodleian Library (London 1898-1903).

rom. = romana.

s. $a_{0}=\sin$ año.

s. fol. $=$ sin foliación.

s. i. $=$ sin impresor.

Sign. = Signatura

s. 1. = sin lugar de impresión.

s. Sign. $=$ sin Signatura.

Vol. = Volumen

5. Catálogo de Incunables

1

AEgidius Romanus, Commentum super libros duos prosteriorum analyticorum Aristotelis cum textu eiusdem (Precede epist. auct. ad Stephanum de Manlay; siguen unos versos).

Venetiis, Simon de Luere, sumpt. Andreae Torresani de Asula, 18 Maii 1500.

128 hoj.- Sign. (1-16) ${ }^{8}$-- fol. [1] 2-128.- Fol.

L. gót.- 3 tam.- 2 col.- 69 lin.- Espac. para inic. con minusc.

GW 7194; Hain 139; Goff A-67; Incun. Hungar. 16; Incun. Ital. 3076; Incun. Polon. 27; Pellechet 84; Catálogo colectivo 51. 
Abundantes notas manuscritas:

Procede de Roma. En f. 1 nota manuscrita: "Collegii Neophyt. Ro", y dos sellos del Colegio. Duplicado.

Encuadernado en AEGIDIUS ROMANUS, Comentaria in octo libros Physicorum Aristotelis. Venetiis, 1502.

\section{2}

AEgrdius Romanus, Commentum super libros duos posteriorum ana1yticorum Aristotelis cum textu eiusdem (Precede epist. auct. ad Stephanum de Manlay; siguen unos versos).

Venetiis, Simon de Luere, sumpt. Andreae Torresani de Asula, 18 Maii 1500.

Es un duplicado del $n$. anterior.

A este ejemplar le faltan los ff. 10 y 15.

Procede de Italia. En f. 7 nota manuscrita: "Fra Giuliano Stronebrosa". En f. 128 nota manuscrita de distinta mano: "Ad usum fratris Iosephi a Stronconio et nonullorum suorum amicorum".

\section{3}

Aegidius Romanus, In Aristotelis de generatione et corruptione Comentarium. (Contiene: Textus Aristotelis de generatione et corruptione cum commentario Aegidii Romani; questiones Marsilii Inghen super libris de generatione et corruptione, emmendatae per Nicoletum Vernia; Tabula; epist. Nicoleti Vernia ad Henricum archiepis. Acheront. et Mater.; quaestio duodecima Nicoleti Vernia).

Venetiis, Otinus de Luna Papiensis, 12 Martii 1500.

116 hoj.- Sign. $a^{6} b^{8}-1^{8} m^{10} n^{8} o^{8} p^{4}$.- s. fol.- Fol.

L. gót.- 3 tam.- 2 col.- 62-69 lín.- Espac. para Inic. con o sin minúsc.

GW 7201; Hain 10782; Goff A-74; Incun. Hungar. 18; Incun. Polon. 35; Proctor 5611; Catálogo colectivo 56; Madrid BN 586; Escorial, Monasterio.

Duplicado. (Véase n. 4).

4

Aegidius Romanus, In Aristotelis de generatione et corruptione Commentarium. (Contiene: Textus Aristotelis de generatione et corruptione cum commentario Aegidii Romani, etc.).

Duplicado del n. anterior. A este ejemplar le faltan los ff. 55, 62 y desde el f. 79 al 87. 
5

Aggidius Romanus, De Regimine Principum. (Precede epist. Oliverii Servii ad Cardin. Georgium archiepisc. Ulixponen; Tabula).

Venetiis, Simon Bevilacqua Papiensis, 9 Iulii 1498.

136 hoj.- Sign. $A^{4} a^{6}-y^{6} .-$ s. fol.- Fol.

L. gr. y rom.- 4 tam.- 2 col.- 44 lín.- Inic. grab. y espac. para Inic. con minúsc.

GW 7219; Hain 109; Goff A-89; Incun. Hungar. 32; Incun. Ital. 3095; Incun. Polon. 47; Pellechet 70; Polain 20; Proctor 5407; Catálogo colectivo 67.

Procede del Convento de S. Agustín de Manila (nota manuscrita de 1828 en f. 1). En el mismo f. nota manuscrita de distinta mano: "Ad usum Fr. Ioannis a $\mathbf{S}$. Nicolao fratrum Eremitarum Ordinis professo, Gen. Inquisitionis Supremi Consilii Qualificatore".

Encuadernado en: AEGIDIUS ROMANUS, Quodlibeta, Venetiis 1502.

$$
\because 6
$$

Albertus de Eyb, Margarita poetica. (Preceden Tabula y Prefacio; sigue Tabula).

Basileae, Iohannes de Amerbach, 1495.

244 hoj. - Sign. $a^{8} b^{8} c^{6} d^{6} e^{8} h^{6} 1^{8} k^{6} 1^{8} m^{6} n^{6} o^{8} p^{6} q^{8} r^{6} t^{6} x^{6} y^{8} z^{6}$ $\mathrm{A}^{8} \mathrm{~B}^{6} \mathrm{C}^{8} \mathrm{D}^{6} \mathrm{E}^{8} \mathrm{~F}^{6} \mathrm{G}^{8} \mathrm{H}^{6} \mathrm{I}^{8} \mathrm{~A}^{6} \mathrm{~B}^{6} \mathrm{C}^{8}$ - s. fol. - Fol.

L. rom. -3 tam. -1 co. $-52-55$ lín. - Espac. para Inic. con minúsc.

GW 9537; Hain-Copinger 6825; Goff E-178; Incun. Ital. 3777; Incun. Polon. 2118; Pellechet 4707; Polain 1451; Catálogo colectivo 1889; Madrid BN 773.

Inic. y calderones con rasgueos er rojo y azul alternando. El rubricador ha escrito' a tinta roja en el f. 1: "Margarita poetica pro usu fratris Georgii Monasterii Furstenfeld professi paratur. Anno 1502". Algunas notas marginales.

$* 7$

Albertus Magnus, S., Sermones notabiles de tempore et de Sanctis. (precede Registrum sermonum).

Ulmae, Iohannes Zainer, [ca. 1478-80]

236 hoj. - s. Sign. - s. fol. - Fol.

L. gót. - 2tam. - 1 col. - 40-41 lín. - Espac. para Inic.

GW 776; Hain 470; Goff A-332; Incun. Ital. 234; Incun. Polon. 124. 
Inic. $y$ calderones en rojo. Abundantes notas manuscritas.

En la gualda nota manuscrita: "Christophor: Pernegger Decan. Maticonens: "1585". En el mismo f. con distinta mano: "Bibliothecae Augustinlanae ad D. V. Schuteranam. Ingolstadil 1634".

*8

Albertus Magnus, S., Sermones notabiles de tempore et de Sanctis. (Precede Registrum Sermonum). Ulmae, Iohannes Zainer [ca. 1478-80]

236 hoj. - s. Sign. - s. fol. - Fol.

L. gót. -2 tam. -1 col. $-40-41$ lín. - Espac. para Inic.

GW. 777; Hain 471; Goff A-333; Incun. Hungar. 74; Incun. Ital. 235; Incun. Polon. 125.

Inic. con rasgueos y calderones en rojo. Abundantes notas marginales. Dañado por el anay, sin afectar al texto.

*9

Pseudo - Albertus Magnus., Compendium Theologicae veritatis cum Tabula Thomae Dorniberg.

Argentinae, [Tohannes Prüss], 1489.

96 hoj. - Sign. $a^{6} b^{8} c^{6}-g^{6} h^{8} 1^{6}-p^{6}$ - - s. fol. - Fol.

L. gót. - 3 tam. - 2 col. - 50 lín. - Espac. para Inic. con o sin minúsc.

GW 603; Hain 442; Goff A-239; Incun. Hungar. 87; Incun. Ital. 173;

Incun. Polon. 142; Proctor 544.

Inic. con rasgueos y calderones en rojo. Al ser encuadernado, fue recortado excesivamente en su margen superior, afectando ligeramente a los títulos. El autor de esta obra parece ser Hugo Ripelin de Argentina.

En f. 2 nota manuscrita: "Convent. Bambergae ord. Prdm".

Encuadernado en: IOHANNES GrITsCh, Quadragessimale 1490.

*10

Pseudo-Albertus Magnus, Compendium Theologicae veritatis cum Tabula Thomae Dorniberg.

Argentinae. [Iohannes Prüss], 1489.

Es un duplicado del anterior.

Inic. con rasgueos y calderones en rojo. El rubricador ha escrito a tinta roja encima del titulo: "Thomas Dorniberg, decretm doctor". $Y$ debajo del título la fecha: "1501". Esta misma fecha la ha repe- 
tido al final del explicit. En el f. 96 ha escrito igualmente: "1501. Explicit totum compendium Theologicae veritatis".

11

Albertus de Saxonia, Comentarius in Posteriora Aristotelis (Siguen una Tabula y epist. Iacobi Baptistae Alovisii ad Carulum Emilianum).

[Venetiis], Bonetus Locatellus Expens. Octaviani Scoti, septimo Idus Martias (9 Martii) 1497.

30 hoj. - Sign. $A^{6}-E^{6}$. - fol. [1] 2-29 [30]. - Fol.

L. gót. - 3 tam. -2 col. - 63-65 lín. - Inic. grab. - Esc. tip.

GW 798; Hain 580; Incun. Hungar. 109; Incun. Ital. 253; Pellechet 389; Proctor 5079; Catálogo colectivo 196.

Encuadernado en: ALBERTUS DE SAXONIA, Quaestiones in Aristotelis libros de coelo et mundo. Venetiis 1492.

12

Albertus de Saxonia, Quaestiones in Aristotelis libros de coelo et mundo, emmendatae per Hieronymum Surianum.

Venetiis, Bonetus Locatellus impens. Octaviani Scoti, nono Kalendas Novembris (24 Octobris) 1492.

51 hoj. - Sign. $\mathrm{A}^{6}-\mathrm{H}^{6} \mathrm{I}^{3}$. - s. fol. - Fol.

L. gót. - 3 tam. - 2 col. - 66 lín. - Inic. grab. - Esc. tip.

GW 796; Hain 576; Goff A-347; Incun. Ital. 251; Incun. Polon. 177;

Pepechet 387 ; Polain 102; Catálogo colectivo 194:

Le falta el f. 1 que tenía el título.

Encuadernado en: Albertus de SAXonia, Commentarius in posteriora Aristotelis, Venetiis 1497.

Alfarabius, véase Aristóteles,

13

AtPhonsus VARGas DE TOLEDo, Lectura in primum librum Sententiarum. (Precede epist. Thomae de Spilimbergo ad Anselmum Montisfalconi, Priorem Generalem O.S.A.; carmen; Tabula y dos carmina).

Venetiis, Paganinus de Paganinis, pridie Kalendas Novembris (31 Octobris) 1490.

173 hoj. - Signn $a^{8} a^{8}-v^{8} x^{5}$ - S. fol. - Fol. 
L. gót. -2 tam. -2 col. -69 lín. - Espac. para inic. con o sin minúsc. - Tinta roja y negra.

GW Hain 876; Goff V-91; Incun. Ital. 406; Incun. Polon. 5527; Pellechet 566 Polain 161; Catálogo colectivo 4681.

Inic. con rasgueos y calderones en rojo.

Al final del Explicit el rubricador fechó su obra: "1592".

14

Alphonsus VARGas DE TOLEDo, Lectura in primum librum Sententiarum. (Precede epíst. Thomae de Spilimbergo ad Anselmum Montisfalconi Priorem Generalem O.S.O., carmen, Tabula y dos carmina).

Venetiis, Paganinus de Paganinis, pridie Kalendas Novembris (31 Octobris) 1490.

Es un duplicado del anterior.

Inic. con rasgueos y calderones en rojo y azul alternando. Los ff. 2-8 están colocados tras los ff. 9-17, y el f. 8 está antes del f. 2.

$* 15$

ANgelus dE Clavasio, Summa angelica de casibus conscientiae (Sigue Tabula).

Norimbergae, Antonius Koberger, V. Kalendas Septembris (28 Augusti) 1488 .

312 hoj. - Sign. $a^{8} b^{6}-z^{6} a a^{6}-z^{6} \quad 22^{6} \quad \partial J^{6} \not 2^{6} \mathrm{AAA}^{8} \mathrm{BBB}^{8}$. - fol. [1] I-CCCX (con errores) [1]. - Fol.

L. gót. -3 tam. -2 col. - 61 lín. - Espac. para inic. con minúsc. GW 1927; Hain-Copinger 5385; Goff A-717; Incun. Hungar. 196; Incun. Ital. 563; Incun. Polon. 553; Pellechet 3816; Proctor 2064; Catálogo colectivo 307; Madrid BN 116.

Inic. con rasgueos y adornos en rojo. En el f. 2 nota manuscrita: "Biblioth: Bamb: 1764".

$* 16$

Angelus Politianus, Opera et alia lectu digna. (Preceden epíst. Aldi Manutii Romani ad Marinum Sannuto y un Index de las obras incluidas; siguen unos versos ad Laurentium Medicem).

Venetiis, Aldus Manutius Romanus, Iulio 1498.

452 hoj. - Sign. $a^{8}-p^{8} q^{10} r^{10} S^{8} t^{8} A^{8}-I^{8} K^{4} L^{8}-P^{8} \cdot Q^{10} R^{10} S^{8} T^{10} V^{6} X^{10}$

$\mathrm{Y}^{10} \mathrm{Z}^{8} \&^{10} \mathrm{aa}^{10} \mathrm{aa}^{8} \mathrm{bb}^{8} \mathrm{cc}^{8}-\mathrm{hh}^{8} \mathrm{ii}^{6} \mathrm{kk}^{10}$. - s. fol. - Fol. 
L. rom. y gr. -2 tam. -1 col. -37 lín. - Espac. para inic. con minúsc.

Hain 13218; Goff P-886; Incun. Hungar. 2808; Incun. Ital. 7952; Incun. Polon. 4530; Polain 3233; Proctor 5567; Catálogo colectivo 3790; Madrid BN 1532; Escorial, Monasterio.

Antoninus Florentinus, S., Summa Theologica. Prima Pars. (Preceden epíst. Francisci Moneliensis; Tabula).

Venetiis, Nicolaus Ienson Gallicus, deciasoctavas Kalendas Ianuarias (15 Decembris) 1479.

254 hoj. - Sign. $a^{10} b^{8}-0^{8} p^{6} q^{8}-t^{8} v^{6} x^{10} y^{8} z^{8} i^{8} y^{8} A^{8}-F^{8} G^{6}$. - s. fol. - Fol.

L. gót. - 2 tam. -2 col. - 55 lín. - Espac. para inic.

GW 2185; Hain 1243; Goff A-872; Incun. Hungar. 250; Incun. Ital. 690; Incun. Polon. 418; Pellechet 878; Proctor 4123.

En f. 1 nota manuscrita: "Loci Capuccinorum Bambergae". Ha sido foliado a mano.

*18

Antoninus Florentinus, S., Summa Theologica. Secunda Pars. (Preceden epist. Francisci Moneliensis; prooemium y Tabula).

Venetiis, Nicolaus Ienson Gallicus, quartas Kalendas Iulias (28 Iunii) 1480 .

322 hoj. - Sign. $a^{10}-d^{10} e^{8}-Z^{8} z^{8} \jmath^{8} \not^{8} \quad A^{8}-L^{8} M^{10} N^{8}$. - s. fol. - Fol.

L. gót. - 3 tam. - 2 col. - 56 lín. - Espac. para inic.

Proctor 4126.

En f. 1 nota manuscrita: "Loci Capuccinorum Bamberge 1638". Ha sido foliado a mano. Abundantes notas marginales.

Antoninus Frorentinus, S., Summa Theologica. Quarta Pars. (Preceden epist. Francisci Moneliensis y Tabula).

Venetiis, Nicolaus Ienson Gallicus, decimas quartas Kalendas Maias (18 Aprilis) 1480.

374 hoj. - Sign. $a^{12} b^{10}-h^{10} i^{8} k^{8} l^{10} \cdot m^{8} n^{10} 0^{8} p^{8} q^{10} r^{8} \cdot s^{10} t^{8} v^{10} x^{8} y^{10} z^{3}$ $2^{10} y^{10} 4^{10} \quad \mathrm{~A}^{10}-\mathrm{K}^{10} \mathrm{~L}^{8} \mathrm{M}^{10} \mathrm{~N}^{10}$. - s. fol. - Fol.

L. gót. - 3 tam. -2 col. - 56 lín. - Espac. para inic. 
Proctor 4124.

En f. 1 nota manuscrita: "Loci Capuccinorum Bamberge 1638".

$\mathrm{Ha}$ sido foliado a mano con errores.

Antoninus Florentinus, S., Summa Theologica. Tertia Pars, subdivisa in duas partes (Precede Tabula).

Venetiis, Leonardus Wild de Ratisbona, 1480.

Pars I: 222 hoj. - Sign. $\mathrm{A}^{10}-\mathrm{N}^{10} \mathrm{O}^{8} \mathrm{P}^{10} \mathbf{Q}^{10} \mathbf{R}^{8} \mathbf{S}^{10} \mathrm{~T}^{8} \quad \mathrm{~V}^{10}-\mathrm{Y}^{10} \mathbf{Z}^{8}$. - Pars. II: 210 hoj. - Sign. $a^{10} b^{8}-f^{8} g^{10} h^{8}-1^{8} m^{10}-y^{10} z^{8}$. - s. fol. - Fol.

L. gót. -2 tam. -2 col. -59 lín. - Espac. para inic. con minúsc. GW 2187; Hain-Copinger 1244; Goff A-873; Incun. Hungar. 251; Incun. Ital. 691; Incun. Polon. 419 ; Pellechet 879; Polain 267; Catálogo colectivo 378; Madrid BN 155.

Inic. y calderones en rojo, azul, amarillo y verde predominando los dos primeros.

*21

Antoninus Florentinus, S., Tabula super totam Summam Theologicam, compilata per Iohanem Molitoris.

[Spirae, Petrus Drach, 1487]

136 hoj. - Sign. $a^{8}-r^{8}$. - s. fol. - Fol.

L. gót. -2 tam. -2 col. -56 lín - Espac. para inic. con o sin minúsc.

GW 2190; Hain 1247 (Tabula); Goff A-876; Incun. Hungar. 254; Incun. Ital. 694; Incun. Polon. 422 (IV); Proctor 2373.

Inic. con rasgueos y calderones en rojo.

Encuadernado en: IoHannes NIVICELLENsis, Concordantiae Bibliae et Canonum totiusque iuris civilis. Basileae 1489.

ARISTóteles, Lat. Rhetorica. (Contiene Declaratio compendiosa Alfarabii super libris rhetoricorum Aristotelis per Lancillotum de Zexlis; Rhetorica Aristotelis translata e graeco in latinum; editio Averrois rhetoricae Aristotelis in latinum cum tabula Lancilloti de Zerlis). 
Venetiis, Philippus Venetus, 22 Iunii 1481.

64 hoj. - Sign. $A^{10} a^{8}-f^{8} g^{6}$. - s. fol. - Fol.

L. rom. - 2 tam. -2 col. - 41 lín. - Espac. para inic. con minúsc.

GW. 2478; Hain 821=Hain-Copinger 1681; Goff A-1046; Incun. Ital. 852; Incun. Polon. 521; Pellechet 512; Catálogo colectivo 494; Madrid BN 202.

En f. 1 nota manuscrita: "Ex usu Corral August.".

Encuadernado en: Declaratio super libris rhetoricorum Aristotelis, MS dorado en 1527 por fr. Fran. de Vadillo, según nota manuscrita en la gualda.

Augustinus, S., De Civitate Dei cum commentariis Thomae Valois et Nicolai Triveth. (Sigue una Tabula).

Basileae, Iohannes Amerbach, Idibus Februariis (13 Februarii) 1489. 268 hoj. - Sign. $a^{10} b^{8}-p^{8} q^{6} r^{8} S^{6} t^{8} v^{6} x^{8} y^{6} A^{8} B^{6} C^{8} D^{6} E^{8} F^{6} G^{8} H^{6} I^{8}$ $\mathrm{K}^{6} \mathrm{~L}^{6} \mathrm{M}^{8} \mathrm{~N}^{6} \mathrm{O}^{8}$. - s. fol. - Fol.

L. gót. -5 tam. -2 y 4 col. - 54 y 65 lín. - Espac. para inic. con minúsc. -1 grab. en madera.

GWI 2887; Hain 2064; Goff A-1243; Incun. Hungar. 376; Incun. Ital. 978; Incun. Polon. 633; Pellechet 1559; Proctor 7578; Catálogo colectivo 89.

Regalado a esta Biblioteca por D. Manuel Huertas, párroco de Moroncillo el 6 de Septiembre de 1884 (nota manuscrita en $f$. 1 y en $f$. 264). En f. 1 nota manuscrita de distinta mano, fechada en 1673 , por la que se ve que perteneció a algún convento de Agustinos y señala que el libro no es propiamente un duplicado y por tanto no se debe permutar. En el mismo f. más abajo nota manuscrita del Abad de los Huertos, Fray Jerónimo de Madrigal, a quien perteneció este incunable. En el f. 264, otro propietario dejó constancia del año en que lo tuvo en su poder a saber, en 1632.

\section{$* 24$}

Augustrnus, S., Opuscula plurima, videlicet, Meditationes, Soliloquia, etc. (Preceden varias Tabulas y unos versos ad Lectorem; siguen Possidius "De Vita et moribus Sancti Augustini" y unos versos).

Argentinae, Martinus Flach, 11 Augusti 1491.

274 hoj. - Sign. $a^{6} b^{8} c^{8} d^{6} e^{8} f^{6} g^{8} i^{8} k^{8} l^{6} m^{8} n^{6} o^{8} p^{6} q^{8} r^{6} s^{8} t^{6} v^{8}$ 
$\mathrm{x}^{6} \mathrm{y}^{8} \mathrm{Z}^{6} \mathrm{~A}^{8} \mathrm{~B}^{6} \mathrm{C}^{8} \mathrm{D}^{6} \mathrm{E}^{8} \mathrm{~F}^{6} \mathrm{G}^{8} \mathrm{H}^{6} \mathrm{I}^{8} \mathrm{~K}^{6} \mathrm{~L}^{6} \mathrm{M}^{8} \mathrm{~N}^{6} \mathrm{O}^{8} \mathrm{P}^{6} \mathrm{Q}^{8}$. - - fol. [6] I-CCLXVII [1]. - Fol.

L. gót. -2 tam. -2 col. -49 lín. - Espac. para inic. minúsc.

GW 2868; Hain-Copinger 1950; Goff A-1221; Incun. Hungar. 363; Incun. Ital. 1019; Incun. Polon. 621; Pellechet 1462; Polain 396; Proctor 691; Catálogo colectivo 80; Madrid BN 15.

En f. 1 nota manuscrita: "Convent. Bambergensis ord. Prdm.".

Augustinus, S., Opuscula plurima, videlicet, Meditationes, Soliloquia, etc. (Preceden varias Tabulas y una nota ad Lectorem; siguen Possidius "De Vita et moribus Sancti Augustini" y unos versos).

Venetiis, Dionysius Bertochus de Bononia, 26 Martii 1491.

350 hoj. - Sign. $a^{10} b^{8}-z^{8} z^{8} \jmath^{8} \not^{8} \quad A^{8} B^{6} C^{8}-R^{8} S^{6}$. - fol. [11] ii-ccc xxxviii [2]. -4 .

L. gót. - 2 tam. -2 col. - 55 lín . Espac. para inic. con o sin minúsc. - Esc. tip. - Tinta roja y negra.

GW 2866; Hain-Copinger 1949; Goff A-1219; Incun. Hungar. 361; Incun. Ital. 1017; Incun. Polon. 620; Pellechet 1461; Polain 4078; Proctor 5277; Catálogo colectivo 78; Madrid BN 14; Cordoba, Cat. 360; Escorial, Agustinos.

Deteriorado por la humedad en el margen inferior derecho sin afectar al texto. Le faltan los dos últimos ff.

En f. 1v. nota manuscrita: "Esta obra fue regalada por el Presbitero D. Marcelino Nava a esta Biblioteca del Colegio de PP. Agustinos de Valladolid". En f. 1 nota manuscrita del revisor del libro por parte de la Inquisición R. Man. Rodriguez, lector jubilado.

\section{*26}

Aulus Flaccus Persius, Satyrae, cum commentario Bartholomaei Fontii. (Precede Prooemium Fontii ad Laurentium Medicem; siguen epist. Fontii ad Franciscum Saxettum y Persii vita).

Venetiis, Dionysius Bertochus et Pelegrinus de Paschalibus, 10 Septembris 1484.

28 hoj. - Sign. $a^{6}-d^{6} e^{4} .-$ s. fol. - Fol.

L. rom. - 3 tam. -1 col. - 51 lín. - Espac. para inic. - Esc. tip.

Hain 12724; Goff P-346; Incun Hungar. 2578; Incun. Polon. 4267; Proctor 4845 .

Abundantes notas manuscritas. Parece proceder de un convento de Agustinos de Alemania, según nota en f. 1, fechada en 1508. 
*27

Aulus Gelluus, Noctes Atticae. (Sigue una Tabula).

Venetiis, Bonetus Locatellus sumpt. Octaviani Scoti, Idibus Novembris (13 Novembris) 1494.

72 hoj. - Sign. $a^{8-1^{8}}$ - fol. [1] 2-72. - Fol.

L. rom. y gr. -3 tam. -1 col. - 62 lín. - Inic. grab. - Esc. tip. Hain 7525; Goff G-125; Incun. Hungar. 1384; Incun. Ital. 4193; Incun. Polon. 2313; Pellechet 5015; Proctor 5057 A; Catálogo colectivo 2090 ; Madrid BN 857.

En f. 1 nota manuscrita: "I. Fr. Beijer, Dillaeburgi 1794". Abundantes notas marginales.

Encuadernado en: Macrobius Aurelius Theodosius, In somnium Scipionis expositiones et Saturnalia. Venetiis 1500.

*28

Azo Portius, Summa super Codice et super Institutionibus cum additionibus aliorum doctorum. (Contiene, Azo Portius, Summa super novem libris Codicis et quattuor Institutionibus ac super libro Novellarum seu Authenticorum. Ioh. Bassianus, Summa ad Pandectam. Placentinus, super tribus libris Codicis. Ioh. Bassianus, Summa super libro Novellarum seu Authenticorum cum additionibus Accursii. Siguen un grab. de S. Antonio Abad y varias Tabulas).

Venetiis, Pholippus Pincius Mantuanus, 1 Iunii 1498.

338 hoj. - Sign. $a^{8}-z^{8} z^{8} j^{8} \not^{8} \quad A^{8}-P^{8} Q^{10}$. - fol. [1] 2-320 [18]. - Fol. L. gót -2 tam. -2 col. -67 lín. - Espac. para inic. con o sin minúsc. - 1 grab. en madera. - Tinta roja y negra.

GW 3147; Hain 2235; Goff. A-1437; Incun. Hungar. 451.

Dos clases de Inic.: unas muy bien hechas en rojo, azul y crema con rasgueos; otras en rojo o azul alternando, al igual que los calderones.

Balbus de Ianua, véase Iohannes balbus de Ianua.

*29

Baptista de Salis, Summa casuum conscientiae dicta Rosella seu Baptistiana. (Precede una nota de presentación. Siguen la Bula de Sixto IV "Etsi Dominici gregis", fechada en Roma, 3 Kalendas Ianuarii (30 Decembris) 1479 y unos versos). 
Norimbergae, Antonius Kogerger, in die $\mathrm{s}$. Tiburtii et Valeriani (14 Aprilis) 1488.

276 hoj. - Sign. $a^{8} b^{6}-z^{6} 2 a^{6}-v^{6} x^{8} y^{8}$. - fol. [1] I-CCLXVII [8]. Fol.

L. gót. 3 tam. -2 col. -61 lin. Espac. para inic. con o sin minúsc. GW 3322; Hain-Copinger $1481=$ Hain 14177; Goff. S-46; Incun. Hungar. 487; Incun. Ital. 1204; Incun. Polon. 782; Polain 3837; Proctor 2063; Catálogo colectivo 598; Escorial, Monasterio; Córdoba, Cat. 505.

En f. 1 nota manuscrita: "Langheim 1641 ".

Bartholonaeus Anglicus, De proprietatibus rerum. (Precede Tabula).

[Heidelbergae, Typ. Lindelbachil $=$ Henricus Knoblochtzer] Kalendas Iunil xil (21 Maii) 1488.

326 hoj. - Sign. (1 $234^{6} a^{8} b^{6}-p^{6} q^{8} r^{6}-t^{6} v^{8} x^{6} g^{6} A^{8} B^{6} C^{6} D^{8} E^{6} F^{6}$ $\mathrm{G}^{6} \mathrm{H}^{6} \mathrm{I}^{8} \mathrm{~K}^{6} \mathrm{~L}^{6}-\mathrm{P}^{6} \mathrm{Q}^{8} \mathrm{R}^{6} \mathrm{~s}$. fol. $\mathrm{T}^{8} \mathrm{~V}^{6} \mathrm{X}^{6} \mathrm{Y}^{8} \mathrm{Z}^{6} \mathrm{AA}^{6} \mathrm{BB}^{8} \mathrm{CC}^{6} \mathrm{DD}^{8}$. Fol.

L. gót. -2 tam. -2 col - 50 lín. - Espac. para inic. con o $\sin$ minúsc.

GW 3411; Hain-Copinger $2507=$ Hain 2508; Goff. B-139; Incun.

Hungar. 506; Incun. Ital. 1257; Incun. Polon. 802; Pellechet 1874; Catálogo colectivo 641; Madrid BN 261.

Los siete primeros ff. están deteriorados en la esquina superior derecha afectando al texto. En f. 1 y 7 nota manuscrita: "Libreria D. Diego".

\section{*31}

Bartolus de SaXoferrato, Lectura super prima Insortiati cum additionibus Alexandri de Imola.

Venetiis, Baptista de Tortis, 18 Decembris 1499.

207 hoj. - Sign. $a^{8}-z^{8} \quad z^{8} j^{8} \not^{8} \quad S$ fol. [1] 2-205 [2]. - Fol.

L. gót. y rom. - 3 tam. - 2, 3 y 4 col. - 75-92 lín. - Espac. para inic. con minúsc. - Esc. tip. - Tinta roja y negra.

GW 3628: Hain 2596; Incun. Ital. 1378.

Abundantes notas manuscritas. En el f. 1 diversos posesores estamparon su firma en el curso del tiempo. 
*32

BARTOLUS DE SAXOFERRATO, Lectura super secunda Insortiati cum additionibus Alexandri de Imola.

Venetiis, Baptista de Tortis, 11 Martii 1500

208 hoj. - Sign. $a^{8}-z^{8} 2^{8} 2^{8} 2^{8}$ fol. [1]. - 2-207 [1]. -Fol.

L. gót. y rom. - 3 tam. - 2, 3, y 4 col. - 71-91 lín. - Espac. para inic. con minúsc. - Tinta roja y negra.

GW 3644; Hain 2596; Incun. Ital. 1392.

A este ejemplar le han sustraído el esc. tip. Diversos posesores estamparon su firma en el $\mathrm{f}$. 1.

Baysio, véase Gurdo de BAysio.

Bebenburgius, véase Lupoldus BAMbergensis.

Bergomensis, véase Iacobus Philippus Bergomensis.

Bernardus di Savona, véase Iohannes Bernardus di Savona.

\section{3}

BIBLIA latina cum glossulis tam marginalibus quam interlinearibus ordinariis una cum Nicolai de Lyra postillis. Sexta Pars. (Contiene Romanos-Apocalipsis; siguen libellus Nicolai de Lyra de perfidia judaica y unos versos de Sebastian Brant ad lectorem).

Basileae, Iohannes Petrus de Langendorff et Iohannes Froben de de Hammelburg, Kalendis Decembris (1 Decembris) 1498.

280 hoj. - Sign. - $a^{8} b^{6} c^{8} d^{6} e^{8} f^{6} g^{8} h^{6} i^{8} k^{6} 1^{8} m^{6} n^{8} o^{6} p^{8} q^{6} r^{8} s^{6} t^{8}$

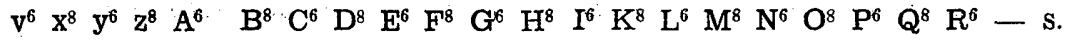
fol. - Fol.

L. gót. -4 tam. -2 col. -56 y 78 lín. - Espac. para inic. con minúsc.

GW 4284; Hain 3172 (Vol. VI); Goff B-609; Incun. Ital. 1693; Incun. Polon. 1040; Pellechet 2351; Polain 681; Catálogo colectivo 858; Madrid BN 347.

34

BrbLia latina cum glossulis tam marginalibus quam interlinearibus ordinariis una cum Nicolai de Lyra postillis. Sexta Pars (Contiene Romanos-Apocalipsis; siguen libellus Nicolai de Lyra de perfidia judaica $\mathrm{y}$ unos versos de Sebastian Brant ad lectorem).

Basileae, Iohannes Petrus de Langendorff et Iohannes Froben de Hammelburg, Kalendis Decembris (1 Decembris) 1498. 
Es un duplicado del $n$. anterior. El último f. está ligeramente danado afectando al texto. En f. 1 nota manuscrita: "Pertinet ad Bibliothecam .Augustinfanam Sarriensem".

\section{5}

Brblia latina cum glossulis tam marginalibus quam interlinearibus ordinariis una cum Nicolai de Lyra postillis. Vol. III. (Contiene Job, Salmos, Proverbios, Eclesiastés, Cantar de los Cantares, Sabiduria y Eclesiástico).

Basileae, Iohannes Petrus de Langendorff, et Iohannes Froben de Hammelburg, ca. 1498-1502.

440 hoj. - Sign. - $a^{8} b^{6} c^{8} d^{6} e^{8} f^{6} g^{8} h^{6} i^{8} k^{6} 1^{8} m^{6} n^{8} o^{6} p^{8} q^{6} r^{8} s^{6}$ $t^{8} v^{6} x^{8} y^{6} z^{8} A^{6} B^{8} C^{6} D^{8} E^{6} F^{8} \quad G^{6} H^{8} I^{6} K^{8} L^{6} M^{8} N^{6} O^{8} P^{6} Q^{8} R^{6} S^{8} T^{6}$ $\mathrm{V}^{8} \mathrm{X}^{6} \mathrm{Y}^{8} \mathrm{Z}^{6} \mathrm{aa}^{8} \mathrm{bb}^{6} \mathrm{cc}^{8} \mathrm{dd}^{6} \mathrm{ee}^{8} \mathrm{ff}^{6} \mathrm{gg}^{8} \mathrm{hh}^{6} \mathrm{il}^{8} \mathrm{kk}^{6} \mathrm{ll}^{8} \mathrm{~mm}^{6} \mathrm{nn}^{8} \mathrm{oo}^{6} \mathrm{pp}^{8}$ $\mathrm{qq}^{6} \mathrm{rr}^{6}$. - fol. [1] 2-440. - Fol.

L. gót. -4 tam. -2 col. -56 y 78 lín. - Inic. grab.

GW 4285; Copinger 1038; Goff B-610; Incun. Ital. 1694; Incun. Polon. 1041.

Le falta el f. 1 (en blanco).

Biblia Germanica. (Contiene Proverbios, Eclesiastés, Cantar de los Cantares, Sabiduria, Eclesiástico, I y II Macabeos, todos los profetas y todo el N.T.).

Strassburgi, [Iohannes (Reinhard) Grüninger], 9 Maii 1485.

456 hoj. - Sign. $a^{8}-v^{8} w^{8} x^{8}-Z^{8} \cdot A^{8}-Z^{8} a a^{8}-k k^{8}$. fol. [1] I-CCCCLXIIII (con errores). - Fol.

L. gót. - 2 tam. - 2 col. - 43 lín. - Espac. para inic. - 21 grab. en madera en colores.

GW 4304; Hain 3138 (Pars II); Goff B-633; Pellechet 2376; Proctor 443.

Inic. con rasgueos en rojo y azul. Falta el f. 1 (en blanco).

*37

Bonaventura, S., Perlustratio in libb. IV Sententiarum. Vol. I. (Preceden epist. Iohannis Bekenhaub ad Nicolaum Tinctorem de Gutzenhausen; un carmen; epist. responsoria Nicolai Tinctoris ad Iohannem Bekenhaub, fechada a 2 de Marzo de 1491; unos versos; sigue Tabula).

Norimbergae, Antonius Koberger, [post 2 Martii 1491]. 
Pars I: 202 hoj. - Sign. $-a^{6}-z^{6} z^{6} g^{6} \mathfrak{x}^{6} a a^{6}-e^{6} \mathrm{ff}^{10} \mathrm{gg}^{6} .-$ s. fol. Fol.

Pars II: 260 hoj. - Sign. $\mathrm{Aa}^{8} \mathrm{Bb}^{6}-\mathrm{ZZ}^{6} \mathrm{Aaa}^{6}-\mathrm{Ttt}^{6} \mathrm{U}^{6}$. - s. fol. Fol.

L. gót. -3 tam. -2 y 3 col. -54 y 64 lín. Espac. para inic. con o sin minúsc.

Hain 3540; Incun. Hungar. 2646; Incun. Polon. 4338; Pellechet 2714; Proctor 2068; Madrid BN 439.

Inic. $y$ calderones en rojo. Abundantes notas manuscritas. Al ejemplar le faltan los ff. 3 y 4 de la primera parte.

*38

Bonaventura, S., Perlustratio in libb. IV Sententiarum. Vol. II. (Siguen epist. Iacobi Wimpfling Sletstantensis ad quoslibet Theol. studiosos, fechada en 1491 y Tabula).

Norimbergae, Antonius Koberger, [post 2 Martii 1491].

Pars. III: 218 hoj. - Sign. $\mathrm{A}^{8} \mathrm{~B}^{6}-\mathrm{Z}^{6} \mathrm{Aa}^{6}-\mathrm{Ll}^{6} \mathrm{Mm}^{8} \mathrm{Nn}^{4}$. - s. fol. Fol.

Pars. IV: 272 hoj. - Sign. $a^{8} b^{8} c^{6}-Z^{6} A^{6}-S^{6} T^{8} V^{8} X^{6}$. - s. fol. - Fol. L. gót. - 3 tam. -1 y 2 col. -54 y 65 lín. - Espac. para Inic. con o sin minúsc.

Hain 3540; Incun. Polon. 4338; Pellechet 2715; Madrid BN 439.

La primera inic. de cada parte en rojo y azul; resto y calderones, en rojo. Abundantes notas manuscritas.

Brack, Wenceslaus, véase Wenceslaus Brack.

*39

Bruno, episc. Herbipolensis, Psalterium ex doctorum dictis collectum.

[Norimbergae], Antonius Koberger, 1497.

172 hoj. - Sign. $a^{8}-x^{8} y^{4} .-$ s. fol. -4 .

L. gót. - 3 tam. -2 col. -52 lín. - Espac. para inic. con minúsc. Hain 4013; Goff B-1057; Incun. Hungar. 2856; Incun. Ital. 8142; Incun. Polon. 4616; Pellechet 3036 ; Polain 919.

En f. 1 hay una nota manuscrita, en la que se dice que el libro perteneció al Obispo de Viena Iohannes Fabri (1530) y después de su muerte fue entregado a la Biblioteca del Colegio de $\mathrm{S}$. Nicolás en 1540 .

Falta el f. 172 que está en blanco.

Caracciolus, véase Robertus Caracciolus de Litto.

Cassiodorus, véase Flavius Magnus Aurelius Cassrodorús. 
Cato, véase Dronyrus Cato

Clavasio, véase angelus de Clavasio.

\section{*40}

Decius Iunius Iuvenaus, Satyrae, cum commentariis Domitii Calderini, Antonii Mancinelli et Georgii Vallae. (Preceden prefacios de los tres comentadores y un Indice).

Norimbergae, Antonius Koberger, 6 Decembris 1497.

198 hoj. - Sign. $A^{8} a^{8}-z^{8} \& z^{6}$ - fol [8] I-CXC (con errores). - Fol.

L. rom y gr. -3 tam. -1 col. $-45-62$ lín. - Espac. para inic. con o sin minúsc.

Hain-Copinger 9711; Goff; J-664; Incun. Hungar. 2004; Incun. Ital. 5601; Incun. Polon. 3322; Proctor 2216.

En f. 1 nota manuscrita: "Dupl. H. V. 31".

Dictionarium graeco-latinum et alia opuscula. (Precede epist. Aldi Manutii Romani studiosis omnibus y contiene siete obras referentes a la gramática).

Venetiis, Aldus Manutius Romanus, mense Decembri 1497.

244 hoj. - Sign. $a^{8}-k^{8} 1^{10} A^{8}-K^{8} L^{10} M^{8} N^{10} O^{8} p^{8}-r^{8} s^{6} t^{8}$. - fol. - Fol. L. rom y gr. -2 tam. -2 y 3 col. $-42-55$ lín. - Espac. para Inic. con 0 sin minúsc.

GW 7814; Hain 6151; Goff. C-960; Incun. Ital. 3255; Incun. Polon. 1803.

Foliado a mano con errores. Abundantes notas manuscritas. En f. 1 nota manuscrita: "Elias Gunskerus. Donavit librum hunc Elias Francke Magister Gunskero mihi, quo sim memor usque in finem". Hay otras notas manuscritas de antiguos posesores.

*42

Drogenes Laertius. Vitae et Sententiae Philosophorum. Lat. (Precede epist. Benedicti Brognoli patriciis Laurentio Georgio et Iacobo Baduario; sigue Tabula).

Venetiis, Philippus Pincius, sumpt. Benedicti Fontana, 22 iunii 1497. 96 hoj. - Sign. $a^{8}-m^{8}$ - fol. [2] iii-xcv [1]. - Fol.

L. rom. -2 tam. -1 col. -46 lín. - Espac. para inic. con o sin minúsc. - Esc. tip.

GW 8384; Hain 6205; Goff D-225; Incun. Hungar 1177; Incun. Ital. 
3464; Incun. Polon. 1919; Pellejchet 4279; Proctor 5317; Catálogo colectivo 1618.

Abundantes notas marginales. Encuadernado en AuLus GELirus, Noctes Atticae, Venetiis 1494.

${ }^{*} 43$

Dronysius Cato, Ethica seu disticha de moribus amplissimo commentario illustrata.

Torquedo Augustae (Ausburg), [Antonius Sorg], die crastina festi Omnium Sanctorum (2 Novembris) 1475.

484 hoj. - s. Sign. - s. fol. - Fol.

L. gót. - 1 tam. - 1 col. - 40 lín. Espac. para inic.

GW 6277; Hain 4711; Goff C-292; Incun. Hungar. 947; Incun. Ital. 2601 ; Incun. Polon. 1468; Pellechet 3429; Proctor 1643.

Primera Inic. miniada. Resto, en rojo, azul o verde. El f. 1 tiene orla por los cuatro márgenes con flores y guirnaldas en varios colores.

$* 44$

Dionystus HaldCarnaseus, Antiquitates Romanae (Lapo Birago interprete). (Precede epist. ad Paulum II Pont. Max.).

Regio (Emilia), Franciscus de Mazalis; 12 Novembris 1498.

226 hoj. - Sign. $a^{8}-z^{8} \&^{8} A^{8}-C^{8} D^{10}$. - fol. [1] ii-ccxxvi. - Fol.

L. rom. -2 tam. -1 col. - 46 lín. - Espac para inic. con o sin minúsc. - Esc. tip.

GW 8424; Hain-Copinger 6240; Goff D-251; Incun. Hungar. 1187; Incun. Ital. 3485; Incun. Polon. 1933; Pellechet 4301; Polain 1313; Proctor 7256; Catálogo colectivo 1690.

Duranti, véase GuILIELmus DuRanti.

Elucidarius Scripturarum, véase Henricus Jerung.

*45

Eusebius Caesariensis, De praeparatione evangelica, Georgio Trapezuntio interprete. (Preceden unos versos, Tabula, epist. Georg. Trapezuntii ad Nicolaum V; siguen epist. Hieronymi Bononii ad Albertum Vonicum Tarvisanum y un carmen Hieronymi Bononii).

Venetiis, Bernardinus Benalius, pridie Kalendas Iunias ( 31 Maii) 1497. 
108 hoj. - Sign. $a^{10} b^{8} c^{6} d^{8}-m^{8} n^{6} o^{6}$. - s. fol. - Fol.

L. rom. - 2 tam. - 1 col. - 45 lín. - Espac. para inic. con o sin minúsc. - 1 grab. en madera (de $\mathbf{S}$. Jerónimo).

GW 9444; Hain-Copinger 6706; Goff E-122; Incun. Hungar 1277; Incun. Ital. 3758; Incun. Polon. 2099; Pellechet 4645; Proctor 4893;

Polain 1433; Catálogo colectivo 1861; Madrid BN 762.

Eyb, véase Albertus DE Eyb.

Fasciculus Temporum, véase Wernerus Rolevinck.

Fenestella, véase Pseudo Luctus Fenestella.

Flavius Magnus Aurelius Cassiodorus, Expositio in Psalterium. (Preceden epist. Iohannis de Lapide ad Ioh. de Amerbach; instructio lectoris; Tabula).

Basileae, Iohannes Amerbach, 1491:

340 hoj. - Sign. $a a^{8} b^{6} c^{8} a^{8} b^{6} c^{8} d^{6} e^{8} f^{6} g^{8} h^{6} i^{8} k^{6} l^{8} m^{6} n^{8}-p^{8} q^{6}$

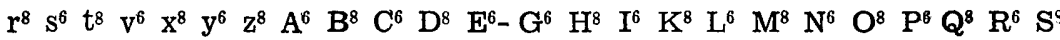
$T^{6} V^{8}-Y^{8}$. - S. fol. Fol.

L. gót. - 5 tam. - 2 col. - 50 lín. - Espac. para inic. con minúsc.

GW 6163; Hain 4574; Goff. C-236; Incun. Hungar. 936; Incun. Ital. 2552; Incun. Polon. 1450; Pellechet 3350; Polain 1020; Protor 7591 ; Catálogo colectivo 1234.

Encuadernado en: Iohannes Gadiensis, Communiloquium seu Summa collationum, Argentinae 1489.

$* 47$

Franciscus Petrarca, Opera. (Precede carmen Sebastiani Brant; contiene Bucolicum carmen; de Vita solitaria; de Remediis utriusque Fortunae; Colloquium trium dierum; de Vera Sapientia; de rebus memorandis; contra medicum objurgantem; epistolae de rebus familiaribus; epistolae sine titulo; epist. ad Charolum IV; de studiorum suorum successibus ad posteritatem; septem psalmi poenitentiales; Epistoma illustrium virorum ad Carrharia; eiusdem Epitomatis Lombardi de Siricho supplementum; Benvenuti de Rambaldis libellus qui Augustalis dicitur; annotatio sententiarum ac materiarum).

Basileae, Iohannes Amerbach, 1496.

389 hoj. - Sign. $A^{8} B^{6} C^{8} A^{8} B^{6}-D^{6} E^{8} a^{8} b^{6} c^{8} d^{6} e^{8} f^{6} g^{10} \quad h^{8} i^{6}-l^{6} m^{3}$ 
$n^{6}-p^{6} q^{8} \cdot a^{8} \quad b^{6} c^{6} \quad F^{4} \cdot a^{11} \quad b^{6} c^{8} d^{6} \quad e^{6} f^{8} g^{8} a a^{8} \quad b b^{10} \quad A^{8} \quad B^{6} \quad C^{8} \quad D^{6} \quad E^{8} \quad F^{6} G^{8}$ $\mathrm{H}^{6} \cdot \mathrm{I}^{8} \mathrm{~K}^{6} \mathrm{~L}^{8} \mathrm{M}^{10} \mathrm{a}^{6} \mathrm{~b}^{10} \mathrm{~A}^{8} \mathrm{~B}^{6} \mathrm{C}^{8}$. - s. fol. - Fol.

L. rom. - 3 tam $-1 \mathrm{col}-49-54$ lín. - Espac. para inic. con miHain 12749; Incun. Hungar. 2587; Incun. Ital. 7564; Goff P-365; Incun. Polon. 4276; Polain 3059; Proctor 7608; Madrid BN 1438.

Inic. con rasgueos en rojo. En la guarda primera, nota manuscrita: "Duplum".

Franciscus Philelphus, Epistolae.

Brixiae, Iacobus Britannicus Brixianus, 7 Maii 1485 .

156 hoj. - Sign. $a^{8} b^{6}-z^{6} \&^{6} 3^{6} B^{6}-$ s. fol. - Fol.

L. rom. y gr. -2 tam. -1 col. -44 lín. - Espac. para inic. con minúsc.

Hain-Copinger 12933; Goff. P-584; Incun. Ital. 3886 (2); Incun. Hungar. 2690; Incun. Polon. 4407; Polain 3134; Proctor 6976; Catálogo colectivo 3660; Madrid BN 1472.

Abundantes notas manuscritas.

*49

Franciscus DE ZabaRelis, Lectura super Clementinis, castigata per Franciscum Moneliensem (Precede epist. Francisci Moneliensis).

Venetiis, Iohannes [Herbort] Magnus de Selgenstat, 28 Ianuarii 1481.

216 hoj. - Sign. $a^{10} b^{8} c^{8} d^{6} e^{6} f^{8}-n^{8} \quad p^{8}-s^{6} \quad t^{6} \quad u^{8}-y^{8} \quad z^{10} \quad A^{8}-D \quad E^{10}-$

s. fol. - Fol.

L. gót. - 2 tam. - 2 col. - 67 lín. - Espac. para inic. - Esc. tip. Hain-Copinger 16252; Goff. Z-4; Incun. Polon. 5727; Catálogo colectivo 4835; Madrid BN 1990; Córdoba, Cat. 486.

Primera inic. miniada con orla en varios colores. Resto de inic. $\mathrm{y}$ calderones, en rojo y azul alternando. Se le ha añadido una hoja como gualda en la que se ha escrito a mano la Tabula. Le falta el último $f$. (en blanco).

*50

Franciscus DE Zabarellis, Lectura super Clementinis.

Venetiis, Iohannes de Forlivio et Gregorius de Gregoriis fratres, 9 Iunii 1487.

186 hoj. - Sign. $a^{10} b^{8}-i^{8} l^{6} m^{6} n^{8}-y^{8} z^{10}$. - s. fol. - Fol. 
L. gót. -2 tam. -2 col. -77 lín. Espac. para inic. con o sin minúsc. - esc. típ.

Hain 16253; Goff. Z-5; Incun. Polon. 5728.

Inic. en rojo o azul con rasgueos. En f. 2 nota manuscrita: "Monasterii B. Mariae V. Fürstenfeldt”.

Gellius, véase Aulus Gellius.

*51

Gratianus, Decretum, cum glossa Bartholomaei Brixiensis.

Venetils, Baptista de Tortis, 30 Martii 1496.

338 hoj. - Sign. $a^{10} b^{8}-z^{8} 2^{8} \cdot 2^{8} 2^{8} A^{8}-Q^{8}$. - [3] 2-335 [1], - Fol.

L. gót. - 3 tam. - 2 y 4 col. - 70-82 lín. - Inic. grab. y espac. para inic con minúsc. - Esc. tip. - Tinta roja y negra.

Hain 7915; Goff. G-388; Incun. Hungar. 1460; Incun. Ital. 4413; Incun. Polon. 2465; Pellechet 5336; Proctor 4654; Catálogo colectivo 1457.

Abundantes notas manuscritas. Procede del Convento de S. Agustín de Manila. Deteriorado en su margen superior afectando ligeramente al texto.

*52

GREgorIus IX, Decretales, cum glossa.

Venetiis, Thomas de Blavis de Alexandria, 22 Decembris 1486.

420 hoj. - Sign $a^{8}-z^{8} z^{8} g^{8} \not^{8} \quad A^{8}-Z^{8} a a^{8} b b^{8} c^{12}$ - s. fol. -4 .

L. gót. -3 tam -2 col. - 54 y. 63 lín. - Inic. grab. y espacios para inic. con minúsc. - Tinta roja y negra. - Esc. tip.

Hain-Copinger 8021; Goff G-463; Incun. Hungar. 1497; Incun. Ital. 4464 ; Incun. Polon. 2522; Proctor 4763; Catálogo colectivo 1466; Madrid BN 668 .

Abundantes notas manuscritas. Tabla de índices a mano al final.

$* 53$

Gregorius IX, Decretales, cum glossa.

Venetiis, Thomas de Blavis de Alexandria, 15 Decembris 1489.

420 hoj. - Sign. $a^{8}-z^{8} z^{8} \cdot y^{8} \not^{8} \quad A^{8}-Z^{8}{a a^{8}}^{b} b^{8} c^{12}$ - fol. [1] 2-419

[1] (con errores). -4 . 
L. gót. -3 tam. -2 col. - 63 lín. - Inic. grab. - Tinta roja y negra.

Hain 8025; Goff G-465; Incun. Hungar. 1498; Incun. Ital. 4468; Incun. Polon. 2524; Catálogo colectivo 1467.

Abundantes notas manuscritas. Tabla de índices a mano en hojas supletorias al principio.

Gritsch, véase Iohannes Gritsch.

GuIdo DE BAysio, Rosarium decretorum, castigatum per Franciscum Moneliensem. (Preceden epist. Francisci Moneliensis y epist. auctoris ad Gerardum episc. Sabinensem).

Venetiis, Iohannes [Herbort] Magnus de Selgenstat pro Iohanne de Colonia et Nicolao Ienson Sociisque, tertio Nonas Aprilis (3 Aprilis) 1481.

416 hoj. - Sign. $a^{10} b^{8} c^{10} d^{10} d^{8}-1^{8} m^{6} \quad n^{6} o^{8}-t^{8} u^{8} v^{8} x^{8} y^{10} z^{8} i^{8} g^{8} z^{8}$ $\mathrm{aa}^{8} \mathrm{bb}^{10} \mathrm{cc}^{10} \mathrm{dd}^{8} \mathrm{dd}^{8} \mathrm{ee}^{10}-\mathrm{gg}^{10} \mathrm{ggg}^{8} \mathrm{hh}^{8} \mathrm{hhh}^{8} \mathrm{ii}^{10} \mathrm{kk}^{8}-\mathrm{mm}^{8} \mathrm{nn}^{10}-\mathrm{qq}^{10} \mathrm{rr}^{8}$ $\mathrm{ss}^{8}$. - s. fol. - Fol.

L. gót. - 2 tam. -2 col. - 66 lín. - Espac. para inic. - Tinta roja y negra. - Esc. tip.

GW 3747; Hain 2717; Goff B-288; Incun. Hungar. 543; Incun. Ital. 4556; Incun. Polon. 876; Pellechet 2014; Polain 1768; Proctor 4677; Catálogo colectivo 713; Madrid BN 290; Cordoba, Cat. 408, 409 у 527. Algunas Inic. en rojo y verde alternando. Calderones en rojo. En la cubierta interior primera, nota manuscrita: "Duplum". Más abajo, un trozo de papel impreso con la leyenda: "Liber collegii societatis Iesu Monachii, Catalogo inscriptus. Anno". En f. 1: "In usum Socie: Iesu Monaci". Más abajo Inc. Typ. $\mathrm{N}=741$. En f. 2: "Aprobatus per R. P. Canisium 1578".

*55

Guilielmus Duranti, Rationale divinorum officiorum (Precede Tabula).

Argentinae, [Iohannes Prüss], 1486.

276 hoj. - Sign. $a^{8}-\mathrm{Z}^{8} \mathrm{~A}^{8}-\mathrm{I}^{8} \mathrm{~K}^{10} \mathrm{~L}^{10}$. - fol. [3] I-CCLXXII [1]. - fol. L. gót. -2 tam. -2 col. - 48 lín. - Espac. para inic. con o sin minúsc.

GW 9131; Hain 6491; Goff D-431; Incun. Hungar. 1239; Incun. Ital. 3636; Incun. Polon. 2025; Pellechet 4508. 
Primera inic. miniada. Resto y calderones en rojo y azul alternando. El f. 4 tiene una orla floreada de varios colores que cubren los márgenes inferior e izquierda. El f. 5v. tiene la misma orla, pero sin colores. El f. 3 está colocado después del f. 4 y el f. 5 después del f. 6.

En f. 1 nota manuscrita: "Duplum". Más abajo con la misma mano: "Pro Conventu Angerensi ad Bibliothecam PP. Franciscan." y un sello con estas letras CA.

\section{*56}

Guilielmus Duranti, Speculum iudiciale cum additionibus Iohannis Andreae et Baldi. Prima Pars.

Venetiis, Georgius Arrivabene [et Paganinus de Paganinis], 12 Iunff 1488.

\section{Segunda Pars.}

Venetiis, Georgius Arrivabene et Paganinus de Paganinis, 10 Septembris 1488.

Pars I: 116 hoj. - Sign. $a^{8}-n^{8} o^{6} p^{6}$. - Pars II: 188 hoj. - Sign. $\mathrm{aa}^{8}-\mathrm{cc}^{8} \mathrm{dd}^{6} \mathrm{ee}^{8}-11^{8} \mathrm{~mm}^{6} \mathrm{nn}^{8}-\mathrm{uu}^{8} \mathrm{xx}^{10} \mathrm{yy}^{8} \mathrm{zz}^{8}: \mathrm{z}^{6}$ - s. fol. - Fol.

L. gót. -2 tam. -2 col. -74 y 98 lín. - Espac. para inic. con 0 sin minúsc. - Tinta roja y negra.

GW 9.158; Hain 6513; Goff D-451. Incun. Ital. 3658; Incun. Polon. 2037; Pellechet 4531; Catálogo colectivo 1791 bis; Córdoba, Cat. 465. Inic. en rojo y azul alternando. En f. 1v. nota manuscrita: "Hic liber est Abbatie florentine". Abundantes notas manuscritas.

\section{*57}

Guthelmus Duranti, Speculum iudiciale cum additionibus Iohannis Andreae et Baldi. Tertia et quarta Pars.

Venetiis, Paganinus de Paganinis et Georgius Arrivabene, 6 Septembris 1488 .

$$
\text { Repertorium Speculi. }
$$

Venetiis, Paganinus de Paganinis et Georgius Arrivabene, octavo Idus Iunii (6 Iunii) 1488.

Pars III et IV: 159 hoj. - $A^{8} B^{8} C^{2} D^{8} E^{8} F^{6} G^{8}-R^{8} S^{10} T^{8} V^{8} X^{5}$. Repertorium: 90 hoj. - Sign. $a^{8}-d^{8} e^{6}-g^{6} h^{8}-m^{8}$. - s. fol. Fol.

L. gót. -2 tam. -2 col. -74 y 98 lín. - Registrum; 70 lín. Espac. para inic. con o sin minúsc.

Inic. en rojo y azul alternando. En f. 1v nota manuscrita: "hinc liber est Abbatie florentine". 
HENRICUS JERUNG, Elucidarius Scripturarum.

Norimbergae, Fridericus Creussner, 6 Iunii 1476.

204 hoj. - s. Sign. - s. fol. - Fol.

L. gót. - 1 tam - 2 col - 48 lín - Espac para Inic.

Hain 9371; Goff J-219; Incun. Ital. 5105 ; Incun: Polon. 3029;

Dos Inic. rubricadas en colores con rasgos finísimos. Resto, en rojo. En f. 1v. nota manuscrita: "Duplum Inc. Typ. Nro. 328".

Hieronymus, S., Commentaria in Bibliam, cura Bernardini Gadoli. (Preceden una relación de opera divi Hieronymi in hoc vol. contenta; una carta de Gregorius de Gregoriis a Hercul. Aestensis, vita $\mathbf{S}$. Hieronymi, descriptio librorum $\mathrm{V}$. et $\mathrm{N}$. Testamenti y una relación (otra) de opera divi Hieronymi in hoc vol. contenta; contiene: liber hebraicarum quaestionum, super Genesim; Oseas, Joel, Amos, Abdias, Jonas, Miqueas, Nahum, Habacuc, Sofonías, Ageo, Zacarías, Malaquias Aquí se halla el colofón y luego sigue Isaías y Lamentaciones).

Venetiis, Iohannes et Gregorius de Gregoriis, 1497.

404 hoj. - Sign. $A^{8}(2,3)^{6}(4,5,6)^{6} a^{8}-c^{8} d^{10} e^{8} f^{6} g^{10} h^{10} i^{8} k^{6} l^{8} m^{8}-$ $\mathrm{u}^{8} \mathrm{X}^{6} \mathrm{y}^{6} \mathrm{~A}^{8}-\mathrm{R}^{8} \mathrm{~S}^{10} \mathrm{~T}^{8}-\mathrm{Z}^{8} \mathrm{AA}^{8} \mathrm{BB}^{6} \mathrm{CC}^{8}$. - s. fol. - Fol.

L. gót. rom. y gr. -4 tam. -1 col - 60 lín. - Inic. grab.

Hain 8581; Goff H-160; Incun. Hungar. 1677; Incun. Ital. 4729; Incun. Polon. 2780; Proctor 4558; Catálogo colectivo 2491; Madrid BN 1028; Córdoba, Cat. 314.

En f. 1 un papel impreso pegado que dice: "Ad Franciscanos Landishuti".

$* 60$

Hreronymus, S., Commentaria in Bibliam, cura Bernardini Gadoli. (Contiene Isaías, Jeremías, y Ezequiel).

Venetiis, Iohannes et Gregorius de Gregoriis, 1497.

326 hoj. - Sgn. $A^{8}-\mathrm{R}^{8} \mathbf{S}^{10} \mathrm{~T}^{8}-\mathrm{Z}^{8} \mathrm{AA}^{8} \mathrm{DD}^{8}-\mathrm{HH}^{8} \mathrm{DDD}^{8} \mathrm{EEE}^{8} \mathrm{FFF}^{6}-\mathrm{HHH}^{6}$ $\mathrm{DDDD}^{6}-\mathrm{GGGG}^{6} \mathrm{HHHH}^{4}$. - s. fol. - Fol.

L. gót. rom y gr. -4 tam. -1 col. - 60 lín. - Inic. grab. y espac. para inic.

Es un duplicado del número anterior, pero con distinta encuadernación. 
Hieronymus, S., Epistolae et Tractatus. (Preceden praefatio, Tabula, vita Hieronymi y unos versos en honor de $\mathbf{S}$. Jerónimo).

Norimbergae, Antonius Koberger, 12 Novembris 1495.

374 hoj. - Sign. $(2,3,4)^{6} \mathrm{a}^{6} \mathrm{~b}^{8}-\mathrm{Z}^{8} \mathrm{~A}^{8}-\mathrm{Y}^{8} \mathrm{Z}^{10}$. - fol. [10] I-CCCLXIIII. Fol.

L. gót. y gr. -3 tam. - 2 col. - 66 lín. - Espac. para inic. con 0 sin minúsc.

Hain 8562; Goff H-174; Incun. Hungar. 1684; Incun. Ital. 4743; Incun. Polon. 2793; Polain 1949; Catálogo colectivo 2499.

Dos Inic. miniadas en oro y varios colores. Resto, en rojo y azul juntamente o bien alternando.

En f. 1: nota manuscrita: "Ad usum FF. Minorum Bambergae ad S. Annam". En f. 1v. nota manuscrita de distinta mano: "Ex largitione venerabilis patris Conradi Knab prioris tunc temporis in Ebrarg; dono datum est volumen Epistolarum omnium B. Hieronymi migi Friderico Dybbaum Herbipolensi. Anno 1547 in praesentia Iorgii Walunff praesenti eodem tempore in Hernsdorff".

*62

Hieronymus, S., Epistolae et Tractatus. Prima et Secunda Pars.

Pars I: [Venetiis, Iohannis Rubeus Vercellensis], 7 Lanuarii (sic) 1496.

Pars II: [Venetiis, Iohannis Rubeus Vercellensis], 12 Iulii 1496.

398 hoj, - Sign. - $(2,3)^{6} \mathrm{a}^{8}-\mathrm{u}^{8} \mathrm{x}^{4} \mathrm{~A}^{8}-\mathrm{Z}^{8} \quad \mathrm{AA}^{8}-\mathrm{CC}^{8} \mathrm{DD}^{6} \quad \mathrm{EE}^{8} \quad \mathrm{FF}^{6}$. fol. [6] 1-392 (con errores). - Fol.

L. rom. y gr. - 3 tam. - 2 col - 62 lín. - Espac. para inic. con minúsc.

Hain-Copinger 8563; Goff H-175; Incun. Hungar. 1685; Incun. Ital. 4745 ; Incun. Polon. 2794 ; Proctor 5141 ; Catálogo colectivo 2500; Madrid BN 1033.

Abundantes notas marginales. En la gualda nota manuscrita de un antiguo posesor fechada en 1654. En f. 2, otra mano: "Pertinet ad Canonicam Castriruppensem".

63

Hieronymus, S., Vitae Sanctorum Patrum.

s. 1.,s.i., s.a.

248 hoj. - Sign. $b^{8}-Z^{8} A^{8}-F^{8}$. - fol. 9-256. - 4.

L. gót: -2 tam. -2 col. -44 lín. Inic. grab. - Grab. en madera. 
Ejemplar muy deteriorado. Faltan los 8 ff. primeros. Al final faltan varios ff. en número indeterminado. Los ocho primeros ff. que tiene, están muy deteriorados; y lo mismo sucede con los 4 últimos ff. finales.

Dado el estado lamentable, nos ha sido imposible determinar de qué edición se trata.

En la gualda nota manuscrita: "Pertinet ad bibliothecam Augustinianam Sarriensem".

IAcobus Magnus, Sophologium.

Lugciuni, Iohannes de Vingle, 26 Iulii 1495.

166 hoj. - Sign. $a^{4} b^{8}-x^{8} y^{2}$. - fol. [1] ii-clvi. - 4.

L. gót. -1 tam. -2 col. - 36-37 lín. - Inic. grab. - Esc. tip. (como portada).

Hain 10479; Goff M-48; Incun. Hungar. 2129; Proctor 8643A.

Algunas notas marginales. En f. 1 (arriba), nota manuscrita: "Collegii Societatis Iesu Cameraci" (Cambrai, Francia). En el mismo f. $\mathrm{y}$ en $\mathrm{f}$. 1v. notas eruditas en francés acerca de las diferentes ediciones de esta obra y un elogio del autor.

*65

Iacobus Philippus Bergomensis, Supplementum Chronicarum. (Preceden varias Tabulas).

Brixiae, Boninus de Boninis de Ragusia, 1 Decembris 1485.

384 hoj. - Sign. $a^{10} b^{10} \quad a a^{2} \quad a^{10} \quad b^{10} \quad c^{8}-t^{8} \quad v^{10} \quad x^{6} \quad y^{8} z^{8} \quad z^{8} \quad g^{8} \quad \not \quad A^{8}-E^{8} \quad F^{6}$

$G^{8}-O^{8} P^{6} Q^{8}-T^{8}$. - fol. [24] 2-358 [1] (con errores). - Fol.

L. gót. - 3 tam. -1 col. - 49 lín. - Espac. para inic.

Hain-Copinger 2806; Incun. Hungar. 1876; Polain 1493; Pellechet 2065 ; Proctor 6965; Catálogo colectivo 2481; Madrid BN 1021; Escorial, agustinos.

Inic. y calderones en rojo. Abundantes notas marginales. Tiene la Tabula generalis antes que las Tabulae speciales, en contra del ejemplar reseñado por Hain. En f. 2. nota manuscrita: "Coll Soc. Jesu Bamb".

*66

Iacobus Philippus Bergomensis, Supplementum Chronicarum. (Precede Tabula).

Venetiis, Bernardus Ricius de Novaria, 15 Madii (sic) 1490. 
273 hoj. - Sign. $(1-6)^{6}(7-12)^{6} a^{8}-z^{8} z^{8} 9^{8} \not^{8} \quad A^{8}-F^{8} G^{5}$. - fol. [12] 1-261. - Fol.

L. gót. - 2 tam. - 1 col. - Inic. grab. y espac. para inic. con minúsc. - Esc. tip. - Grab. en madera.

Hain-Copinger 2808; Goff J-211; Incun. Hungar. 1878; Incun. Ital. 5078; Incun. Polon. 2969; Pellechet 2067; Polain 1495; Proctor 4954; Catálogo colectivo 2483; Madrid BN 1023.

El ejemplar antes de llegar a esta biblioteca pasó por el colegio de los PP. Agustinos de Zaragoza (sello en f. 2). Le falta el f. 1 (en blanco). Los ff. correspondientes a la signatura $\not$ se encuentran antes de los correspondientes a la signatura 2. Algunas notas manuscritas.

IOHANNES XXI, Summulae logicae cum commento Iohannis Versoris. (Precede epist. Petri de Sancto Iohanne ad Iohannem Pardum; sigue tractatus parvarum logicalium).

Venetiis, Hermanus Lichtenstein Coloniensis, vii nonas Martii (sic) 1488.

140 hoj. - Sign. $a^{10} \cdot b^{8}-k^{8} 1^{6}-s^{6} t^{10}$. - fol. [2] 3-140. - Fol.

L. gót. -3 tam. -2 col. - 53-68 lín. - Espac. para inic. con o sin minúsc.

Hain-Reichling 8691; Goff J-232; Incun. Ital. 5220; Incun. Polon. 5580; Catálogo colectivo 2683; Madrid BN 1103.

En f. 1: varias notas "ex-libris" de antiguos posesores, que son todos dominicos. En el mismo f. nota manuscrita: "Conventus Rottwilani Fratrum Praedicatorum".

Iohannes Balbus de IANuA, Summa quae vocatur Catholicon.

Norimbergae, Antonius Koberger, duodecimo kalendas Septembris (21 Augusti) 1486.

22. hoj. - Sign. $a^{7} b^{8}-h^{8} i^{6} k^{8} 1^{8} m^{6}-z^{6} a a^{6}-Z^{6} A^{6} a^{6} d^{6} E^{7} .-$ s. fol. Fol.

L. gót. -2 tam. -2 col. - 70 lín. - Espac. para inic. con o sin minúsc.

GW 3192; Hain-Copinger 2258; Goff B-28: Incun. Polon. 736; Polain 1716; Catálogo colectivo 586.

Primera inic. miniada en oro, azul, rojo y verde. Resto, en rojo o azul con rasgueos. Calderones en rojo. 
69

IoHannes Bernardus dr Savona, Vocabulista Ecclesiastico.

Mediolani, Leonardus Pachel, 5 Novembris 1495.

84 hoj. - Sign. $a^{8}-k^{8} 1^{4}$. s. fol. -8.

L. gót. - 1 tam. -1 col. - 32 lín. - Inic. grab. y espac. para inic. con minúsc.

Goff B-358.

En la cubierta interior nota manuscrita: "Ex libris R. P. Herculis Balconil". En el mismo lugar (más abajo): "Comprado en Roma en 1962. B. Rano O.S.A. 25.10.70".

*70

IohanNes Duns Scotus, In quartum librum Setentiarum.

[Argentinae], s. i., 1474.

286 hoj: - s. Sign. - s. fol. - Fol:

L. gót. - 1 tam. - 2 col. - 51 lín. - Espac. para inic.

GW 9084 ; Hain 6430; Incun. Hungar. 1217; Goff D-377; Incun. Polon. 2000; Proctor 342.

Inic. con rasgueos y calderones en rojo y azul alternando.Faltan los dos primeros ff. Dañado al principio y al final por el anay sin afectar al texto.

*71

Iohannes Gallensis, Summa Collationum sive Communiloquium ad omne genus humanum (Precede Tabula).

Argentinae, [Typ. Iordani=G. Husner], in die sancti Urbani (25 Maii) 1489.

80 hoj. - Sign. $a^{8} b^{6}-n^{6}$. - s. fol. - Fol.

L. gót. -2 tam. -2 col. -52 lín. - Espac. para inic. con o sin minúsc.

Hain 7444; Goff J-332; Incun. Hungar. 1811; Incun. Ital. 5269; Incun. Polon. 3132; Polain 2282; Proctor 649; Catálogo colectivo, 2542. Encuadernado en Marcus Aurelius Casstodorus, Expositio in Psalterium, Basileae 1491.

$* 72$

IOHANNES GRITSCH, Quadragesimale. (precede Tabula).

[Argentinae, Typ. Vitas Patrum] in die Sanctae Agathae virg. (5 Februarii) 1484. 
266 hoj. -2 tam. -2 col. - 50 lín. Espac. para inic. con o sin minúsc.

Hain-Copinger 8070; Goff G-500; Incun. Hungar. 1519; incun. Polon. 2554; Proctor 420.

Inic. con rasgueos $\mathrm{y}$ calderones en rojo. En f. 2 nota manuscrita: "Sum Joan. Murmani D.S.Th.". Más abajo: "Nunc loci Capuccinorum Bambergae 1643" Faltan los ff. 1 y 266 (ambos en blanco).

\section{*73}

Iohannes Gritsch, Quadragesimale. (Precede Tabula).

[Argentinae, Typ. Iordani=G. Husner] 1490.

239 hoj. - Sign. $a^{8}-d^{8} e^{6} f^{8} g^{6} h^{8} i^{6} k^{8} l^{6} m^{8} n^{6} o^{8} p^{6} q^{8} r^{6} s^{8} t^{6} v^{8} x^{6}$ $y^{8} z^{6} A^{8} B^{6} C^{8} D^{6}-L^{6} M^{5}$. - s. fol. - Fol.

L. gót. -2 tam. -2 col. - 52 lín. - Espac. para inic. con o minúsc.

Hain 8075; Goff G-504; Incun. Hungar. 1522; Incun. Ital. 4497; Incun. Polon. 2560. Proctor 659.

Inic. con rasgueos y calderones en rojo. Algunas notas manuscritas. Encuadrenado en Pseudo-Albertus Magnus, Compendium theologicae veritatis. Argentinae 1489 (Véase n. 9).

IoHANNes Marchesinus, véase Mammotrectus; super Bibliam.

$* 74$

IOHANNES MELBER DE Geroltzhoffen, Vocabularius praedicantium seu Variloquus.

[Argentinae, Typ. Iordani-G. Husner, ca. 1497].

172 hoj. - Sign. $a^{8}-e^{8} f^{4} \cdot g^{8} h^{8} i^{4} k^{8} 1^{4} m^{8}-0^{8} p^{4} q^{8}-t^{8} v^{4} x^{8}-z^{8} z^{8} .-s$.

fol. $-4 .{ }^{\circ}$.

L. gót. - 2 tam. - 1 col. - 36 lín. - Espac. para inic. con minúsc.

Hain 11034; Goff M-469; Incun. Ital. 6350; Incun. Polon. 3700; Proctor 645 .

En f. 1 nota manuscrita: "pro Conventu fm. Minorum".

*75

IohanNes Nivicellensis, Concordantiae Bibliae et Canonum totiusque iuris civilis.

Basileae [Michael Furter (?)], último vo (sic) Ianuarii 1489. 
76 hoj. - Sign. $\mathrm{a}^{8} \mathrm{~b}^{6}-1^{6} \mathrm{~m}^{8}$. - s. fol. - Fol.

L. gót. -2 tam. -2 col. -44 lín. - Mayúsc. como inic. y espac. para inic.

Hain 9417; Goff J-382; Incun. Hungar. 1939; Incun. Ital. 5331; Incun. Polon. 3192; Proctor 7577; Polain 2297; Catálogo colectivo 2577. Inic. con rasgueos y calderones en rojo.

Encuadernado en Antonius Frorentinus, Tabula super totam Summam Theologicam, compillata per Iohannem Molitorem. Spirae 1487. (Véase n. 21).

Iohannes Picus de Mrrandula, Opera philosophica, theologica, cum eius Vita. (Contiene epist. Francisci Pici Mirandulae ad Ludovicum Mariam Sforziae; Iohannis Pici vita per Iohannem Franciscum conscripta; Cypriani epis. Carthag. de ligno crucis carmen; Heptaplus de opere sex dierum Geneseos; Deprecatoria ad Deum elegiaco carmine; apologia tredecim quaestionum; Breve Alex. VI Papae ad Iohannem Picum; tractatus de ente et uno; oratio quaedam elegantissima; epistolae plures; Disputationum adversus Astrologos libri duodecim prooemium operis contra Astrologos; dos cartas más y Tabula).

Venetiis, Bernardinus de Vitalibus Venetus, 9 octobris et 14 Augusti 1498.

262 hoj. - Sign. $A^{10} A^{6} B^{6} C^{4}-E^{4} F^{6}-Q^{6} R^{4} S^{6}-X^{6} Y^{4} Z^{6} \&^{6} a^{6}-S^{4} t^{4} u^{6} y^{4}$. s. fol. - Fol.

L. rom. - 2 tam. - 1 col. - 44 lín. - Inic. grab. y espac. para inic. con 0 sin minúsc.

Hain-Copinger 12993; Goff P-634; Incun. Ital. 7733; Incun. Hungar. 2718; Incun. Polon 4432; Polain 3144; Proctor 5526; Catálogo colectivo 3687; Madrid BN 1482.

En cubierta interior, nota manuscrita: "Ad Conventum Monacensem Ordinis Eremitarum S. Augustini 1731". En la gualda primera: "Duplum".

$* 77$

IoHANNes Reuchlin, Capnio dictus, Vocabularius breviloquus. (Precede Ars disphtongandi Guarini Veronensis; dialogus de arte punctuandi; tractatus utilis de acentu).

Norimbergae, Antonius Koberger, 12 Iulii 1498.

294 hoj. - Sign. $(1,2,3,4,)^{6} \mathrm{a}^{8}-\mathrm{Z}^{8} \mathrm{~A}^{8}-\mathrm{N}^{8}$. - S. fol. $-4 .{ }^{\circ}$. 
L. gót. - 2 tam. -2 col. - 57 lín. - Espac. para Inic con minúsc. Copinger 6283; Incun. Ital. 8341; Polain 3345.

IOHANNES TORTELlIUS ARETINUS, Commentariorum Grammaticorum de Ortographia dictionum e Graecis tractarum opus. (Precede prooemium ad Nicolaum V Pont. Max.; siguen epist. Hieronymi Bonini ad Constantinum Robeganum, eiusdem carmen in primi impressoris commendationem).

Vicentiae, Hermanus Lichtenstein Coloniensis, die ultima (31) Octobris, 1480 .

316 hoj. - Sign. $A^{9} B^{8}-O^{8} P^{6} a^{8}-r^{8} s^{8} s^{8}-z^{8} \&^{5}$. - s. fol. Fol.

L. rom y gr. -2 tam. -1 col. - 46 lín. - Espac. para inic. con o sin minúsc.

Hain 15567; Goff T-398; Incun. Polon. 5380; Catálogo colectivo 4548; Madrid BN 1880.

IOHANNES TORTELLIUS ARETINUS, Commentariorum Grammaticorum de Ortographia dictionum e Graecis tractarum opus. (Precede epist. Pyrrhi Pincii Mantuani ad Franciscum Capello patritium Venetum; Prooemium ad Nicolaum V Pont. Max.; sigue Tractatus de Ortographia Georgii Valla).

Venetiis, Philippus Pincius, pridie Idus Aprilis (12 Aprilis) 1493.

188. hoj. $\rightarrow$ Sign. $a^{8}-y^{8} Z^{6} \&^{6}$. - s. fol. - Fol.

L. rom. y gr.. -2 tam. -1 col. -61 lín. - Espac. para inic con 0 sin minúsc.

Hain-Copinger 15572: = Hain 15577; Goff T-401; Incun. Polon. 5383; Polain 3796; Catálogo colectivo 4551; Madrid BN 1882.

*80

Iohannes de Turrecremata, Expositio super toto Psalterio. (Precede una dedicatoria a Pío II).

Augustae Vindelicorum, Iohannes Schüssler, pridie nonas Maias (6 Maii) 1472.

134 hoj. - s. Sign. - s. fol. - Fol.

L. gót. - 1 tam. -1 col. 35 lín. - Espac. para inic.

Hain 15696; Goff T-519; Incun. Hungar. 3386; Protor 1596. 
Dos inic. en rojo y verde con rasgos finísimos a toda plana.

Resto de inic. y calderones, en rojo.

En f. 1 nota manuscrita: "Monasterii B. V. Deiparae in Diessen".

*81

Iunianus MaIus Partenopaeus, De priscorum proprietate verborum. (Sigue epist. auctoris ad Hernricum archiepisc. Acheront.).

Tarvisiae, Bernardus de Colonia, 1477.

330 hoj. - Sign. $a^{10}-c^{10} d^{8}-f^{8} g^{10}-r^{10} S^{8} S^{10} t^{8} y^{10} x^{8} y^{10} a a^{12} b b^{10}-11^{10}$. s. fol. - Fol.

L. gót. - 1 tam. - 2 col. - 51 lín. - Espac. para inic.

Hain 10540; Goff M-96; Incun. Hungar. 2136; Incun. Polon. 3527; Incun. Ital. 6037; Polain 2575; Proctor 6483; Catálogo colectivo 2974. Inic. con rasgueos y calderones, en rojo. En f. 2 nota manuscrita: "Ex Bibliotheca Reverendissimi Episcopi Augustani".

Iustinus, véase MaRcus Iunianus Iustinus.

Iuvenalis, véase Decius Iunius Iuvenalis.

Jerung, véase Henricus Jerung.

$* 82$

LaCtantius Frrminus, Opera. (Contiene Tabula; errata Lactantii collecta et exarata per Antonium Raudensem; quomodo legendi sunt libri Lactantii; praefatio ad Constantinum imperatorem; de divinis Institutionibus libri septem; de Ira Dei ad Donatum; de opificio Dei et formatione hominis ad Demetrianum; Carmina de Foenice; de die resurrectionis dominicae; Nephytomon; Tertullianus, Apologeticus adversus Gentes; Tabula Tertulliani).

Venetiis, Bonetus Locatellus, expens. Octaviani Scoti, quinto Idus Octobris (11 Octobris) 1494.

90 hoj. - Sign. $a^{8}-k^{8} 1^{10}$. - fol [1] 2-90. - Fol.

L. rom. y gr. -3 tam. -1 col. - 63 lín. Inic. grab. - Esc. tip. Hain 9817; Goff L-12; Incun. Hungar. 2022; Incun. Polon. 3343; Incun. Ital. 5629; Polain 2424; Proctor 5056; Catálogo colectivo 2724; Madrid BN 1121.

Algunas notas marginales. 
$* 83$

LeONARDUS DE UTINo, Sermones aurei de Sanctis.

[Coloniae, Ulricus Zell] 1473.

471 hoj. - s. Sign. - s. fol. - Fol.

L. gót. - 1 tam. -2 col. - 36 lín. - Espac. para inic.

Hain-Copinger 16128; Goff L-151; Incun. Hungar. 2051; Incun. Polon. 3406; Polain 2479; Proctor 881.

Inic. de dos clases: unas en rojo y azul con rasgueos a todo o medio folio. El resto, en rojo o azul alternando. Calderones en rojo. En f. 1 nota manuscrita: "Liber domus sororum Collismariae in Coeffeldia". El ejemplar tiene los 25 ff., que Hain-Copinger pone al final, al comienzo del libro.

*84

Leonardus de Utino, Sermones aurei de Sancti. (Precede Tabula sermonum).

Norimbergae, Antonius Koberger, undecimo Kalendas Februarii (22 Ianuarii) 1478.

211 hoj. - s. Sign. - fol. [1] I-CCX. - Fol.

L. gót. -2 tam. -2 col. - 50 lín. - Espac. para inic.

Hain-Copinger 16134; Goff L-159; Incun. Hungar. 2056; Incun. Ital. 5741; Incun. Polon. 3412; Polain 2481; Proctor 1982.

Inic. en rojo $\mathrm{y}$ azul junto o bien alternando. Calderones en rojo y azul alternando.

En f. 1 nota manuscrita: "Liber Iohannis Streber 1519". Más abajo, otra mano: "Langheim 1673 ".

Livius, véase Trusus Livrus.

Lucanus, véase Marcus annaeus Lucanus.

*85

Lucas de Burgo, Summa de Arithmetica, Geometria, Proportioni et Proportionalita. (Preceden dicatio auct. ad Patritium Venetum Marcum; duo carmina; epist. auct. ad Guidum Ubaldum, italiana et lingua latina exarata).

Vinegia, Paganino di Paganini da Brescia, 10, 12 Novembre 1494. 308 hoj. - Sign. $(1,2,3,4)^{8} \mathrm{a}^{8}-\mathrm{Z}^{8} i^{8} \mathrm{j}^{8} \mathrm{z}^{8} \mathrm{AA}^{14} \mathrm{~A}^{8}-\mathrm{H}^{8} \mathrm{I}^{6} \mathrm{~K}^{6}:-$ fol [9] 2-224; 1-76. - Fol.

L. gót. - 3 tam. -1 col. - 59 lín. - Inic. grab. y espac. para inic. con o sin minúsc. - Grab. en madera geométricos. 
Hain-Copinger (add.) 4105; Gôf L-315; Incun. Ital. 7133; Incun. Polon. 3457; Pellechet 3060; Catálogo colectivo 2B66; Mađirid BN 1173 (parte de la obra).

En f. 1 nota manuscrita: "Iste liber est fratris Jo: Baptistae de Carmignolis emporiensis Ord. Erem. S. Augustini".

*86

Ludolphus de Saxonia, Meditationes Vitae Iesu Christi.

[Argentinae, Typ. Vitas Patrum] in die Sancti Lucae (18 Octobris) 148.3

329 hoj. - Sign. $A^{9} B^{8}-E^{8} F^{10} \cdot G^{9} \cdot H^{8}-K^{8} \cdot L^{10} M^{8}-S^{8} T^{6} U^{8} X^{6} a^{8}-t^{8} V^{7}$. - s. fol. - Fol.

L. gót. - 2 tam. - 2 col. 63 lín. - Espac. para inic.

Hain 10293; Incun. Hungar. 2109; Goff L-343; Incun. Polon. 3477; Polain 2534; Catálogo colectivo 2878; Madrid BN 1181.

La primera inic. en rojo y azul con rasgueos a media columna. Resto, en rojo y azul alternando. Calderones en rojo.

Muy deteriorado por el anay, pero sin afectar grandemente al texto.

$* 87$

Ludolphus DE SAxonia, Meditationes Vitae Iesu Christi. (Preceden una Tabula y un prólogo y sigue otra Tabula).

Norimbergae, Antonius Koberger, vigilia Assumptionis Virginis Mariae (14 Augusti) 1495.

312 hoj. - Sign. $A^{8}-Z^{8}: a^{8}-q^{8}$. - s. fol. - Fol.

L. gót. - 3 tam. - 2 col - 66 lín. - Espac. para inic. con o sin minúsc.

Hain 10296; Incun. Hungar. 2112; Goff L-346; Incun. Polon. 3478; Proctor 2100; Catálogo colectivo 2882; Madrid BN 1183.

Inic. y calderones en rojo. En f. 1 nota manuscritas de posesores del libro, una de ellas fechada en 1522.

\section{*88}

Pseudo-Lucius Fenestella, De Romanorum Magistratibus. (Sigue Albericus Philosophus de imaginibus deorum; Tabula).

[Medioloni, Leonhard Pachel et Ulrichus Scinzenzeler, non post 7 Novembris 1478].

56 hoj. - Sign. $a^{8}-g^{8}$. - s. fol $-4 .^{\circ}$.

L. rom. - 2 tam. - 1 col. - 22-25 lín. - Espac. para inic. con minúsc. 
Hain 6959; Goff F-63; Incun. Hungar 1305; Incun. Ital. 3815 (?); Pellechet 4755.

Foliación y enumeración de los capítulos, manuscrita. El autor parece ser Andrea Domenico Fiocco.

$* 89$

Pseudo-Lucius Fenestelia, De Romanorum Magistratibus.

[Venetiis, Philippus Petri, ca. 1472-1477].

42 hoj. - 1. Sign. - s. fol. - 4. ${ }^{\circ}$.

L. rom. -2 tam. -1 col. - 23 lín. - Espac. para inic. con minúsc.

Hain 6958; Incun. Ital. 3812; Pellechet 4754.

Algunas notas manuscritas.

$* 90$

LUMEN animae seu liber moralitatum. Edit. Matthias Farinator. (Preceden Prologus et Tabula).

[Argentinae, Typ. Legendae Aureae], sexta feria post Laetare (22 Martii) 1482.

274 hoj. - s. Sign. - s. fol. - Fol.

L. gót. - 3 tam. -2 col. 43 lín. - Espac. para inic. con o sin minúsc.

Hain 10333; Goff L-396; Incun. Hungar. 2033; Incun. Polon. 3492; Pellechet 4749; Polain 1468; Proctor 413; Catálogo colectivo 2903.

Algunas inic. en rojo, azul y verde; Resto, en rojo, así como los calderones. En 1 nota manuscrita: "Duuplum"; en f. 2: "Ad PP. Franciscanos Novenburgi. Bibliotheca".

*91

LUPOLDUS BAMBERGENSIS, Germanorum veterum principum zelus et fervor in christianam religionem, etc. (Preceden carmen Sebastiani Brant ad Iohannem Dalburgium; epist. Iacobi Wimpfelingii ad Fr. Camerarium de Dalburgio).

Basileae, Iohannes Bergmann de Olpe, Idibus Mail (15 Maii) 1497.

28 hoj. - Sign. $a^{8} b^{6} c^{6} d^{8}$. - fol [1] II-XXVII [1]. - Fol.

L. rom. - 4 tam. - 1 col. - 41 lín. - Espac. para inic. con minúsc.

Hain 2725; Goff L-399; Incun: Hungar. 2067; Incun. Ital. 5748; In- 
cun. Polon. 3494; Pellechet 2015; Polain 2486; Catálogo colectivo 2904 ; Escorial, Monasterio.

Lyra, véase Nicolaus de Lyra.

*92

Macrobius Aurelius Theodostus, In somnium Scipionis expositiones et Saturnalia.

Venetiis, Philippus Pincius Mantuanus, 29 Octobris 1500.

36 y 86 hoj. - Sign. $a^{6}-d^{6}$ y $A^{6}-N^{6} O^{8}$. - fol. [2] III-XXXVI y ILXXXVI. - Fol.

L. rom. y gr. -3 tam. -1 col. - 45 lin. - Primera inic. grab.. resto, espac. para inic. con minúsc. -8 grab. en madera.

Hain-Copinger 10340; Goff M-13; Incun. Ital. 5928; Incun. Hungar. 2122; Incun. Polon. 3500; Polain 2553; Proctor 5325; Catálogo colectivo 2911; Madrid BN 1197.

A veces los espacios dejados para las palabras griegas han sido escritos a mano. Abundantes notas manuscritas.

Encuadernado en: Aulus Gellius, Noctes Atticae, Venetiis 1494. (Véase n. 27).

*93

Mammotrectus super Bibliam. Edit. Iohannes Marchesinus.

Argentinae, [Martinus Flach] 1487.

317 hoj. - Sign. $a^{8}-Z^{8} A^{8}-Q^{8} R^{5}$ - fol. [32] I-CCLXXXV. - 4. ${ }^{\circ}$.

L. gót. - 2 tam. -2 col. - 37 lín. - Espac. para inic. con minúsc.

Hain-Copinger 10566; Goff M-248; Incun. Hungar. 2175; Incun. Ital. 6154; Incun. Polon. 3595; Polain 2607; Proctor 674.

Inic. y calderones con rasgueos, en rojo. En f. 2 nota manuscrita: "Conventus Bambergensis Ordinis Praedicatorum".

*94

Mammotrectus super Bibliam. Edit. Iohannes Marchesinus. (Precede Tabula).

[Argentinae, Typ. Paludani (?) vel Vitas Patrum (?), ca. 1485].

172 hoj. - Sign. 14 ff. (sin Sign.) $a^{8-t^{8}} v^{6} .-$ fol. [17] 1-CLV. Fol.

L. gót. - 2 tam. - 2 col. - 46 lín. - Espac. para inic. con minúsc. 
Hain 10553; Goff M-246; Incun. Hungar. 2174; Incun. Polon. 3593; Polain 2603.

Inic. $\mathrm{y}$ calderones en rojo y azul alternando. En f. 2 nota manuscrita: Loci Capuccinorum Bambergae 1639".

MARChesinus, véase Mammotrectus super Bibliam.

\section{5}

Marcus Annaeus Lucanus, Pharsalia, cum Omniboni Vicentini commento. (Precede vita Lucani ex Commentario Antiquiss).

Venetiis, Nicolaus Battibonus Alexandrinus, tertio Idus Maii (13 Maii) 1486.

186 hoj. - Sign. $a^{8}-x^{8} y^{6} z^{6} \&^{6}$. - s. fol. - Fol.

L. rom. - 3 tam. -1 col. -57 lín. - Espac. para inic con minúse. Hain 10238; Goff L-302; Incun. Hungar. 2097; Incun. Ital. 5819; Incun. Polon. 3453; Proctor 5106; Catálogo colectivo 2858.

En la gualda, nota manuscrita: "PP. Filipinos de Villadolid".

*96

Marcus Fabius Quintilianus, Institutiones oratoriae, cum annotationibus Raphaelis Regii. (Precede Tabula; sigue notitia de Quintiliano.

Venetiis, Bonetus Locatellus sumpt. Octaviani Scoti, XVI Kalendas Sextilis (17 Iulii) 1493.

206 hoj. -2 ff. (sin Sign.) $a^{8}-Z^{8} \&^{8} J^{6} R^{6}-$ s. fol. Fol.

L. rom. y gr. -2 tam. -1 y 2 col. -46 y 61 lin. - Inic. grab. y espac. para inic. con minúsc. - Esc. tip.

Hain-Copinger (add.) $13654=13652$; Goff Q-29; Incun. Hungar. 2885; Incun. Ital. 8264; Incun. Polon. 4655; Proctor 5045; Catálogo colectivo 3889; Madrid BN 1578.

Abundantes notas manuscritas. En el f. último, originalmente en blanco, una mano ha transcrito la epist. Campani ad Antonium Moretum que aparece en ediciones posteriores de esta obra.

*97

Marcus Iunianus Iustinus, Epitome historiarum Trogi Pompeii.

Venetiis, Philippus Condam Petri, 12 Decembris 1479.

104 hoj. - Sign. $a^{10} b^{8} c^{8} d^{6} e^{8}-1^{8} m^{6} n^{10}$. s. fol. - Fol.

L. rom. - 2 tam. - 1 col. - 36 lin. Espac. para inic. con o sin minúsc. 
Hain-Copinger 9651; Goff J-618; Incun. Hungar. 1988; Incun. Ital. 5556; Incun. Polon. 3307; Proctor 4274; Catálogo colectivo 2694.

Abundantes notas marginales.

98

Marcus Valerius Martialis, Epigrammatum libri, cum comentario Domitii Calderini. (Preceden epist. Domitii Calderini ad Iohannem Franciscum; Calderini versus; epist. Calderini ad Laurentium Medicem; vita Martialis; epist. Plinii II ad Cornelium Priscum; sigue epist. Calderini ad Corelium; epist. M. Lucidi Phospori).

Venetiis, Baptista de Tortis, 17 Iulii 1485.

172 hoj. - Sign. $a^{8}-u^{8} x^{6} y^{6}$. - s. fol. - Fol.

L. rom. -3 tam. -1 y 2 col. $-41-63$ lin. - Espac. para inic. Esc. tip.

Hain-Copinger 10819; Goff M-308; Incun. Hungar. 2190; Incun. Ital. 6227; Incun. Polon. 3612 ; Proctor 4631; Catálogo colectivo 3020 ; Madrid BN 1232.

En cubierta interior: "pertenece a esta Biblioteca".

Marsilius ab Inghen, véase Aegidius Romanus.

Martialis, véase Marcus Aurelius Martialis.

$* 99$

Martinus Polonus, Margarita decreti seu Tabula Martiniana.

Argentinae, [Typ. Iordani=Ge. Husner], in die sabbati post Pentecostem (13 Iunii) 1489.

106 hoj. - Sign. $a^{8} b^{6}-q^{6} r^{8}$ - s. fol. - Fol.

L. gót. -2 tam. -2 col. -52 lín. - Espac. para inic. con o sin minúsc.

Hain-Copinger 10845; Goff M-325; Incun. Hungar. 2202; Incun. Ital. 6241; Incun. Polon. 3624; Polain 2525; Catálogo colectivo 3073.

Falta el f. 106 (en blanco).

En los ff. 2 y 105 hay una nota manuscrita, en la que se dice que el libro perteneció al Obispo de Viena Iohannes Fabri (1530) y después de su muerte fue entregado a la Biblioteca del Colegio de $\mathbf{S}$. Nicolás en 1540. (Véase el n. 39). 


\section{*100}

MARYRoLogIUm quod et Viola Sancorum inscribitur.

[Argentinae, Iohannes Prüss] 8 Februarii 1499.

134 hoj. - Sign. $(1,2,3,4,5){ }^{10} a^{8} b^{6} c^{6} d^{4} e^{4} f^{8} g^{8} h^{4} i^{4} k^{8} l^{8} m^{6} n^{6} o^{4}$ $\mathrm{p}^{4} \mathrm{q}^{8} \mathrm{r}^{4} \mathrm{~s}^{4} \mathrm{t}^{6} \mathrm{v}^{6} \mathrm{x}^{8}$. - fol. [10] I-CXXIII [1]. - 4.

L. gót. -2 tam. -1 col. - 35 lín. - Mayúsc. como inic. -2 grab. en madera.

Hain 10873; Incun. Hungar. 2216; Incun. Ital. 6261; Goff M-339; Incun. Polon. 3638; Polain 2635; Proctor 552; Catálogo colectivo 3082.

Algunas notas manuscritas.

Maius Partenopaeus, véase Iuntanus Maius Partenopaeus.

Melber de Geroltzhoffen, véase Iohannes Melber de Geroltzhoffen.

$* 101$

MEFfret, Sermones de tempore. Pars Estivalis. (Sigue Tabula).

[Basileae, Nicolaus Kessler, 24 Maii 1488].

273 hoj. - Sign. aa $a^{8}-\mathrm{kk}^{8} 11^{10} \mathrm{~mm}^{10} \mathrm{nn}^{8} \mathrm{oo}^{10} \mathrm{pp}^{8}-\mathrm{rr}^{8} \mathrm{ssS}^{8} \mathrm{sS}^{6} \mathrm{tt}^{8} \mathrm{vv}^{8} \mathrm{ww}^{\mathrm{s}}$ $\mathrm{xx}^{8} \mathrm{yy}^{8} \mathrm{ZZ}^{6} \mathrm{AA}^{8} \mathrm{BB}^{8} \mathrm{CC}^{6} \mathrm{EE}^{8} \mathrm{FF}^{6}-\mathrm{KHK}^{6} \mathrm{LL}^{5}$. - s. fol. - Fol.

L. gót. - 2 tam. - col. 57 lín. Espac. para inic.

Hain 11006 (VoI. II); Goff M-446; Incun. Hungar. 2250; Incun. Ital. 6335; Incun. Polon. 3687; Polain 2658.

Primera inic. miniada en oro, rojo, verde y morado. Resto y calderones, en rojo.

En f. 1 nota manuscrita: "Ex Bibliotheca Canonicorum Regularium S. Augustini. Trebonae 1718".

*102

Nicolaus DE LyRa, Postilla super Psalterium et Prophetas cum additionibus Pauli Episcopi Burgensis ac replicis magistri Matthiae Dorinck.

s. 1., s. i., s. a.

466 hoj. - s. Sign. - s. fol. - Fol.

L. gót. - 3 tam. -2 col. - 72-73 lín. - Espac. para inic. - 13 grab. en madera.

Como no lo hemos visto en nigún repertorio, hacemosde él una descripción más amplia:

f. 1: en blanco; f. 2: Postilla venerabilis fratris Nicolai de// Lyra 
sup psalterium feliciter incipit/f ( ) Ropheta magnus surrexit in / nobis Luce. vij. Qzuis liber // psalmoru apud hebreos inter // ... $f$. 5: lin. 9: Incipit prologus beati // hiero. psbri inpsalteriu. $/ /$... f. 156v. col. 2. lín. 45: Explicit postilla supra librum psalmoru edita a fra $N /$ tre Nicolao de lyra ex ordine minoru socre theologie // doctore excellentissimo. f. 157, en blanco. f. 158: postilla: Incipit prologus in Esaiam pphetam $N /()$ Emo cum pphetas etc. Iste plogus diui $/ /$ dit... Textus: Incipit prologus i Esa /// iam prophetam.// ( ) Emo cum pro//phetas versib ${ }^{9} / /$ viderit ee descri // ... f. 466 , col. 2, lín. 53: Postilla fratris Nicholai de lyra cum additionib ${ }^{9} / /$ Pauli epi burg. ac replicis magistri Mathei doringk $/ /$ eiusde ordinis sup malachiam pphetam finit.

Inic. y calderones en rojo. El rubricador ha puesto después del $e x-$ plicit, la fecha, que no es clara. Es 1484 o 1488.

*103

Nicolaus Salicetus, Antidotarius animae.

Argentinae, Iohannes Reynardus (Grüninger), Idus Iulii septimas (9 Iulii) 1489.

152 hoj. - Sign. $a a^{8}-b b^{8} a^{8}-p^{8} A^{8} B^{8}$. - fol. [16] 1-120 [16]. -8.

L. gót. - 2 tam. -2 col. - 34 lín. Espac. para inic. - Tinta roja y negra.

Hain-Copinger (Add.) 14156; Goff S-35.

Inic. en rojo. En f. 1 nota manuscrita: "Convent. Friburg. Ord. Praed.". Al ser encuadernado ha sido cortado excesivamente en el margen superior de tal modo que a veces no es visible la foliación y en el f. 34 la línea superior ha sido cortada a medio.

Ovidius, vẻase Publius Ovidrus Naso.

104

Paulus Venetus, Comentaria in libros posteriores Aristotelis.

Venetiis, Theodorus de Rheinsburch et Reinaldus de Novimagio, 14 Iunii, 1477.

180 hoj. - Sign. $a^{8}-0^{8} p^{6} q^{8}-y^{8} z^{6}$. s. fol. - Fol.

L. gót. - 1 tam. -2 col. -50 lín. - Espac. para inic. còn o sin minúsc.

Hain-Copinger 12511; Goff P-212; Incun. Ital. 7334; Incun. Polon. 4199; Polain 3017; Catálogo colectivo 3479; Madrid BN 1406.

La primera inic. no ha sido rubricada. La inic. de la segunda parte, rubricada en varios colores con rasgos finos. Resto de inic. y calderones en rojo. Se ignora la procedencia. 
105

Paulus Venetus, Comentaria in libros posteriores Aristotelis.

Venetiis, Bonetus Locatellus, mand. Octaviani Scoti, decimo kalendas ianuarias (23 Decembris) 1491.

96 hoj. - Sign. aa ${ }^{8}-\mathrm{mm}^{8}$. - s. fol. - Fol.

L. gót. - 3 tam. -2 col. - 63 y 69 lín. - Inic. grab. - Esc. tip. Hain-Copinger 12513; Goff P-215; Incun. Hungar. 2538; Incun. Ital. 7337; Incun. Polon. 4202; Proctor 5030; Catálogo colectivo 3482 ; Madrid BN 1408.

Abundantes notas marginales. Procedencia ignorada.

\section{6}

Paulus Venetus, Summulae, seu Logicae Institutiones.

Venetiis, Guilielmus Tridinensis de Monteferrato, tertio nonas Septembris ( 3 Septembris) 1488

48 hoj. - Sign. $a^{8}-f^{8}$ - s. fol. $-4 .^{\circ}$

L. gót. -1 tam. -2 col. -49 lín. - Inic. grab. y mayúsc. como inic.

Hain-Copinger 12501; Goff P-227; Incun. Hungar. 2542; Catálogo colectivo 3494 .

Algunas notas marginales. En f. 1 notas manuscritas en italiano.

Persius, véase Aulus Flaccus Persius.

Petrarca, véase Franciscus Petrarca.

- Petrus Hispanus, véase IoHannes XXI.

*107

Petrus Lombardus, Sententiarum libri. (Preceden prologus y Tabula; sigue otra Tabula).

Basileae, Nicolaus Kessler, Octavo nonas Martii (sic) 1486.

229 hoj. - Sign. $a^{8} b^{6} c^{8} d^{6} e^{8} f^{6} g^{8} h^{6} i^{8} k^{8} l^{6} m^{8} \cdot n^{6}-r^{6} s^{8} t^{6} v^{8} x^{6}-z^{6}$

$\mathrm{A}^{8} \mathrm{~B}^{6} \mathrm{C}^{8} \mathrm{D}^{6} \mathrm{E}^{8} \mathrm{~F}^{6} \mathrm{G}^{6} \mathrm{H}^{8} \mathrm{I}^{8} \mathrm{~K}^{9}$. - S. fol. - Fol.

Hain 10190; Goff P-484; Incun. Hungar. 2640; Incun. Ital. 7637; Incun. Polon. 4336; Polain 3117; Proctor 7654; Catálogo colectivo 3624. Inic. y calderones en rojo. Faltan los ff. 118 y 158 (en blanco). En f. 2 nota manuscrita: "Ex Testamento Reverendiss. D. Frid. Förnerii Sufraganei Bamb. Collegii Socie. Jesu Bamb. 10 Ianua. 1631". 
*108

Petrus Lombardus, Sententiarum libri, cum conclusionibus Henrici Gorichem. (Preceden Prologus et Tabula; sigue otra Tabula).

Basileae, Nicolaus Kessler, Decimo Kalendas Iunii (23 Maii) 1487. 292 hoj. - Sign. $a^{10} b^{6} c^{8}-t^{8} A^{8}-O^{8} P^{6} Q^{6} R^{8} S^{8}$. - s. fol. - Fol.

L. gót. -6 tam. -2 col. -54 y 68 lín. - Espac. para inic. con o sin minúsc. - Esc. tip.

Hain 10194; Goff P-490; Incun. Hungar. 2642; Incun. Polon. 4341; Polain 3119 ; Proctor 7660 ; Catálogo colectivo 3626 ; Córdoba, Catálogo 463.

Inic. con rasgueos y calderones en rojo. En f. 1 nota manuscrita: "Fr. Mm. Bambergensium ad S. Anna".

*109

Petrus Lombardus, Sententiarum libri cum conclusionibus Henrici Gorichem. (Preceden Prologus et Tabula); sigue otra Tabula).

Basileae, Nicolaus Kessler, Decimo Kalendas Iunii (23 Maii) 1487. Es un duplicado del n. anterior. En f. 1 nota manuscrita: "FF. Min. Bamb. ad S. Annam. En cubierta interior, otra mano: "Ioan: Oruis Canonicus Ecclae. Aschaffenburgan. "Numerosos versos en latín en cubierta interior y en f. 1.

*110

Petrus Lombardus, Sententiarum libri cum conclusionibus Henrici Gorichen. (Preceden Prologus et Tabula; sigue varii articuli erronei in quibus Magister communiter non tenetur y una Tabula fere omnium sententiarum).

Basileae, Nicolaus Kessler, Decimo Kalendas Martii (20 Februarii) 1498.

256 hoj. - Sign. $a^{6}-\mathrm{r}^{6} \mathrm{SS}^{6} \mathrm{~S}^{6} \mathrm{SS}^{6} \mathrm{t}^{4} \mathrm{~A}^{8} \mathrm{~B}^{6}-\mathrm{F}^{6} \mathrm{FG}^{6} \mathrm{G}^{6}-\mathrm{H}^{6} \mathrm{I}^{8} \quad \mathrm{~K}^{6}-\mathrm{P}^{6} \mathrm{Q}^{8} \mathrm{R}^{6}$ $\mathbf{S}^{8} \mathbf{T}^{10}$ - S. fol. - Fol.

L. gót. - 5 tam. -2 col. - 50-70 lín. - Espac. para inic. - Esc. tip.

Hain 10198; Goff P-496; Incun. Hungar. 2650; Incun. Ital. 7646; Incun. Polon. 4347; Proctor 7693.

Inic. y calderones en rojo y azul.

En f. 1 nota manuscrita: "Liber Conventus Lipsensis". Más abajo: "Hic liber spectat ad fratrem Heinrichum Reuus Ordinis Predicatorum Conventus Lipsensis ad s. Paulum". En el mismo f. con otra mano: "nunc. Monast. Banz.". 
Philelphus, véase Franciscus Philelphus.

Picus de Mrrandula, véase Iohannes Picus de Mrrandula.

Polimianus, véase Angelus Politianus.

*111

Prisctanus Grammaticus, Opera, cum commentario Iohannis de Aingre. (Precede dicatio ad Iulianum Consulem; contiene De octo partibus orationis cum commento Iohannis de Aingre; de constitutione dictionum; de duodecim carminibus; de accentibus; de numeris, ponderibus et mensuris; de praeexcitamentis rhetoricae ex Hermogene translatis; opusculum de comicorum versuum ratione; commentarium Rufini de metris comicis; de declinationibus pronominum ac verborum libellus; de orbis situ ex Dionysio translato; editio recognita a Benedicto Brugnolo).

Venetiis, Georgius de Arrivabene, pridie nonas Decembris (4 Decembris) 1488.

346 hoj. - Sign. $a^{8}-v^{8} x^{6} y^{8} z^{8} \&^{8} j^{8} R^{-8} a a^{8}-e e^{8} f f^{6} A^{8}-L^{8} M^{6}$. - s. fol. Fol.

L. rom. y gr. -3 tam. -2 col. -43 y 60 lin. - Espac. para inic. con o sin minúsc.

Hain 13361; Goff P-968; Incun. Hungar. 2831; Incun. Ital. 8053; Incun. Polon. 4572; Proctor 4914; Catálogo colectivo 3834.

*112

Publius Ovidius Naso, Metamorphoseon libri, cum commentario Raphaelis Regii. (Preceden privilegium Raphaele Regio concessum, nota ad Lectorem, praefatio Raphaelis Regii ad Franciscum Gonzagam, Index; siguen dos cartas de Rafael Regio).

Venetiis, Bernardinus Venalius, [post 5 septembris, 1493].

167 hoj. - Sign. $A^{6} a^{8}-t^{8} v^{6} x^{3}$. - s. fol. - Fol.

L. rom. y gr. -3 tam. -1 y 2 col. -45 y 58 lín. - Espac. para inic. con minúsc. -1 grab. en madera.

Hain 12170=Hain 12155; Goff O-189; Incun. Ital. 7122; Incun. Polon. 4100; Catálogo colectivo 3384.

Inic. con rasgueos en rojo.

Quintillianus, véase Marcus Fabius Quintrutanus. 
$* 113$

RABBI SAMUEL, Epistola ad Isaac seu rationes breves ad reprobandos Judaeorum errores, de arabico in latinum translata per Alphonsium Bonihominis, hispanum, ord. Praed. (Sigue epist. quam misit Poncius Pilatus Tiberio Imperatori Romano).

[In Sancto Urso, Iohannes de Reno. ca. 1477-80 (?)].

Hain 14263; cfr. Copinger III, p. 560; Incun. Hungar. 3021; Polain 3425; Proctor 7149; Catálogo colectivo 4050; Madrid BN 1637.

*114

Radulphus Strodus, Consequentiae, cum commento Alexandri Sermonetae et Declarationibus Gaetani de Thienis. (Siguen Dubia Pauli Pergulensis, Obligationes Syrodi, Consequentiae Ricardi de Ferebrich, expositio Gaetani super easdem).

Venetiis, Bonetus Locatellus, sumpt. Octaviani Scoti, tertio Idus Februarii (11 Februarii) 1493.

130 hoj. - Sign. $a^{8}-p^{8} q^{10}-$ s. fol. $-4 .^{\circ}$.

L. gót. -3 tam. -2 col. -39 y 50 lín. - Inic. grab. - Esc. tip. Hain 15098; Goff S-802; Incun. Ital. 9178.

Abundantes notas marginales. Le falta el último $f$. (en blanco).

Reuchlin, véase IOANNES REuchuIN.

${ }^{*} 115$

Robertus Caracciolus de Litio, Sermones Quadragesimales de poenitentia. (Sigue Tabula sermonum).

Venetiis, Andreas Torresanus de Asula, 5 Kalendas Octobris (27 Septembris) 1488.

192 hoj. - Sign $a^{8}-z^{8} z^{2}$ - fol. [1] $2-191$ [1]. $-4 .^{\circ}$

GW 6080 ; Hain 4439 ; Goff $\mathrm{C}-160$; Incun. Hungar. 913 ; Incun. Ital. 2465; Incun. Polon. 1415; Pellechet 3258; Polain 988; Proctor 4719; Catálogo colectivo 1209.

Falta el f. 1 y el último (ambos en blanco). En f. 2 nota manuscrita tachada, otra más abajo borrada y pegada encima un papel impreso con esta leyenda:" "Biblioteca Francesco Agosti".

ROLEVINCK, véase WERNERUS RoLEVINCK.

Salicetus, véase Nicolaus Salicetus.

Scotus, véase Iohannes Duns Scotus.

Strodus, véase Radulphus Strodus. 
*116

SUIDAs, Lexicon graecum. (Preceden un diálogo en griego, unos versos en latín de Antonius Motta a Demetrius Chalcondylas y una carta de Iohannes Sallandus a Demetrius Chalcondylas y Ad lectorem).

Mediolani, Demetrius Chalcondylas, Iohannes Bissolus et Benedictus Mangius, 15 Novembris 1499.

516 hoj. - Sign. $\alpha^{\gamma}-(1)^{8} \alpha \alpha \alpha^{8} \lambda \lambda \lambda^{8} A^{8} Q^{8} A A 8 \quad \Delta \Delta^{8}-\mathrm{EE}^{6} z^{6}-$ s. fol. - Fol.

L. gr. y rom. -2 tam. -1 col. -45 lín. - Espac. para inic. con o sin minúsc. - Esc. tip.

Hain-Copinger 15135; Goff S-829; Incun. Hungar. 3161; Incun. Ital. 9789; Incun. Polon. 5134; Polain 3631; Proctor 6077; Catálogo colectivo 4301; Madrid BN 1750; Córdoma, Cat. 515; Escorial, Monasterio. Algunas notas marginales.

\section{7}

Thomas de Aquino, S., Scriptum super I libro Sententiarum. (Precede una Tabula y sigue otra).

Venetiis, Bonetus Locatellus, sumpt. Octaviani Scoti, quinto nonas Octobris ( 3 Octobris) 1498.

149 hoj. - Sign. $A^{8}-S^{8} T^{5}$. - fol. 1-149 - Fol.

L. gót. -4 tam. -2 col. - 52 y 65 lín. - Inic. grab.

Hain 1475; Goff T-162; Incun. Hungar. 3282 ; Incun. Ital. 7650 ; Incun. Polon. 5280; Pellechet 1065 (I); Polain 3730; Proctor 5091; Catálogo colectivo 4470; Madrid BN 1837.

En la gualda nota manuscrita: "Pertenece a la Librería de PP. Filipinos de Valladolid". Le faltan los dos ff. primeros y del f. 149 ha sido sustraido el esc. tip.

Encuadernado en: THowas DE AquiNo, Scriptum super II libro Sententiarum, Venetiis, 1498. (véase el n: 118).

Thomas DE Aquino, Scriptum super II libro Sententiarum. (Precede una Tabula y sigue otra).

Venetiis, Bonetus Locatellus, expens. Octaviani Scoti, undecimo Kalendas Ianuarii (22 Decembris) 1498.

158 hoj. - Sign. $a^{8}-t^{8} v^{6}$ - fol. [1] 2-158. - Fol.

L. gót. -4 tam. -2 col. -52 y 63 lín. - Inic. grab.

Hain-Copinger 1478; Goff T-165; Incun. Hungar. 3284; Incun. Ital. 
7652; Incun. Polon. 5283; Pellechet 1065 (II); Polain 3732; Proctor 5096; Catálogo colectivo 4473.

Faltan los ff: 1 (que tenía el título) el f. 60 , y el f. 158 (que tenía el esc. tip. y parte de la Tabula).

Encuadernado en $T$ Homas dE AQuino, Scriptum super I libro Sentententiarum. Venetiis 1498.

${ }^{*} 119$

Thomas de Aquino, S., Opuscula. (Preceden Index omnium opusculorum; Praefatio Antonii Pizamani in vitam S. Thomae; legenda seu vita eximii Doctoris; Praefatio Antonii Pizamani in opuscula; sigue Tabula).

Venetiis, Hermanus Lichtenstein Coloniensis, vii Idus Septembris (7 Septembris) 1490.

430 hoj. - $\mathrm{aa}^{12} \mathrm{a}^{8}-\mathrm{v}^{8} \mathrm{x}^{12} \mathrm{~A}^{8}-\mathrm{Z}^{8} \mathrm{AA}^{8}-\mathrm{GG}^{8} \mathrm{HH}^{12}$. - s. fol. - 4 .

L. gót. - 2 tam. -2 col. -55 lín. - Espac. para inic. con minúsc. Hain-Copinger 1541; Goff T-258; Incun. Hungar. 3223; Incun. Polon. 5382; Pellechet 1093; Polain 3713; Proctor 4793; Catálogo colectivo 4420; Madrid BN 1815.

Inic. en rojo. En f. 1 nota manuscrita: "Iste liber attinet venali Caenobio Sancti Nicolai Induden Sancti Benedicti".

$* 120$

Thomas de Aquino, S., Summa Theologica. Secunda Pars. secundae partis. (Precede Tabula).

Venetiis, Iohannes de Colonia Agrippinensis et Iohannes Manthen de Gherretzem, XIII Kalendas Augusti (20 Iulii) 1480.

286 hoj. - Sign. - $(1,2,3,4)^{8} a^{8}-z^{8} A^{8}-L^{8} M^{6}$. - s. fol. Fol.

L. gót. -2 tam. -2 col. $-56-57$ lín. - Espac. para inic.

Hain 1464; Goff T-216; Incun. Polon. 5316; Pellechet 1054; Polain 3755; Catálogo colectivo 4494; Córdoba, Cat. 353.

Primera Inic. en rojo y azul con rasgueos. Resto y calderones en rojo y azul alternando.

*121

Thomas DE ARgentina, Scripta super quatuor libros sententiarum. Vol. I. (Preceden epist. Pallas Spangel ad Martinum Flach y varias Tabulas).

Argentinae, Martinus Flach, 1490. 
284 hoj. - Sign. $(1,2,3,4,5)^{8} a^{10} b^{8}-e^{8} f^{6} \cdot g^{8}-k^{8} l^{6} m^{8} \cdot n^{6} \cdot o^{8} p^{6} q^{8} r^{6} s^{8}$ $\mathrm{t}^{6}-\mathrm{y}^{6} \mathrm{z}^{8} \mathrm{~A}^{8}-\mathrm{E}^{8} \mathrm{G}^{6} \mathrm{H}^{8} \mathrm{I}^{6} \mathrm{~K}^{6} \mathrm{~L}^{8} \mathrm{M}^{6} \mathrm{~N}^{8} \mathrm{O}^{6} \mathrm{P}^{8}$. - s. fol. - Fol.

L. gót. -2 tam. -2 col -53 lín. Espac. para inic. con minúsc. Copinger 603; Goff T-343; Incun. Hungar. 3305; Incun. Polon. 5351; Pellechet 1164; Polain 3766; Catálogo colectivo 4400; Madrid BN 1806 ; Escorial, Agustinos.

Inic. con rasgueos y calderones en rojo. En f. 2 nota manuscrita: "Conventus Bambergensis".

*122

Thomas de ARgentina, Scripta super quatuor libros Sententiarum. Vol. II. (Siguen unos versos).

Argentinae, Martinus Flach, 1490.

284 hoj. - Sign. aa ${ }^{10} \mathrm{bb}^{6}-\mathrm{dd}^{6} \mathrm{ee}^{8} \mathrm{ff}^{6} \mathrm{gg}^{8} \mathrm{hh}^{6}-11^{6} \mathrm{AA}^{8} \mathrm{BB}^{6} \mathrm{CC}^{6} \mathrm{DD}^{8} \mathrm{EE}^{8}$

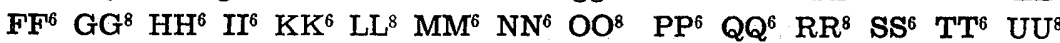
$\mathrm{XX}^{6} \mathrm{YY}^{6} \mathrm{ZZ}^{8} \mathrm{AAA}^{6} \mathrm{BBB}^{6} \mathrm{CCC}^{9} \mathrm{DDD}^{6} \mathrm{EEE}^{6} \mathrm{FFF}^{8} \mathrm{GGG}^{6} \mathrm{HHH}^{6} \mathrm{III}^{6}$. s. fol. - Fol.

L. gót. -2 tam. -2 col. - 53 lín. - Espac. para inic. con minúsc. Copinger 603; Goff T-343; Incun. Hungar. 3305; Incun. Polon. 5351; Pellechet 1764; Polain 3766; Proctor 690; Catálogo colectivo 4400; Madrid BN 1806; Escorial, Agustinos.

Inic. con rasgueos y calderones en rojo. En f. 2 nota manuscrita: "Ad Bibliothecam Monasterii s. Mich: Arch: Bamb. O.S.B.".

\section{*123}

Trtus Lrvius, Historiae Romanae Decades. (Preceden epist. Iohannis episc. Aleriensis ad Paulus II, L. Flori epitoma decadum y capita operum).

Taryisii, Iohannes [Rubeus] Vercellensis, 1485. 256 hoj. - Sign. $a^{10} b^{7} c^{9} d^{8}-n^{8} a^{8} \mathrm{ij}^{8} k^{6} l^{6} A^{8}-G^{8} H^{10}$. - s. fol. Fol.

L. rom. -2 tam. -1 col. -59 lín. Espac. para inic.

Hain-Copinger 10136; Goff L-244; Incun. Ital. 5777; Incun. Polon. 3429; Polain 2499; Incun. Hungar. 2077; Proctor 6499; Catálogo colectivo 2819; Madrid $\mathrm{BN} 1146$.

Inic. en rojo y azul, pero sólo en la primera parte del libro. Abundantes notas marginales.

Tortellius, véase Iohannes Tortellius ARetinus.

TURReCremata, véase Iohannes DE TuRrecremata. 
Utino, véase Leonardus de Utmino.

Vargas de Toledo, véase alphonsus Vargas de Toledo.

Vocabulartus, véase IoHanNes Reuchinn.

*124

WeNCESLaUs Brack, Vocabularius; Isidorus Hispalensis, Etimologiarum liber $\mathrm{X}$, excerptus modo alphabetico; Wenceslaus Brack, de Verbis; de modo epistolandi; Hugonis a $\mathbf{S}$. Victore Didascalicon.

[Coloniae], s. i. in vigilia Simonis et Iudae (27 Octobris) 1483.

98 hoj. - Sign. $a^{8-c^{8}} d^{10} e^{10} f^{8} g^{10} h^{6}-1^{6} m^{10}$. - s. fol. - Fol.

L. gót. -2 tam. -2 col. - 49 lín. - Espac. para inic. con o sin minúsc.

Hain 3700.

Faltan los dos primeros ff. que contenían la Tabula. En f. 3 nota manuscrita: "Bibliothecae Weissenau".

$* 125$

[Wernerus Rolevinck], Fasciculus temporum. (Precede Tabula).

[Argentinae, Iohannes Prüss, ca. 1490].

96 hoj. - Sign. $(1,2,3)^{6} \mathrm{~A}^{8} \mathrm{~B}^{6}-\mathrm{O}^{6} \mathrm{P}^{4}$ - fol. [6] I-XC. Fol.

L. gót. - 3 tam. -1 y 3 col. 50 lín. - Espac. para inic. con o sin minúsc. - grab. en madera.

Hain 6916; Goff R-276; Incun. Ital. 8425; Incun. Polon. 4800; Proctor 563 ; Madrid BN 1620 .

Zabarellis, véase Franciscus de Zabarellis.

Constantino Mielgo, OSA.

Estudio Teológico Agustiniano

VALLADOLID 